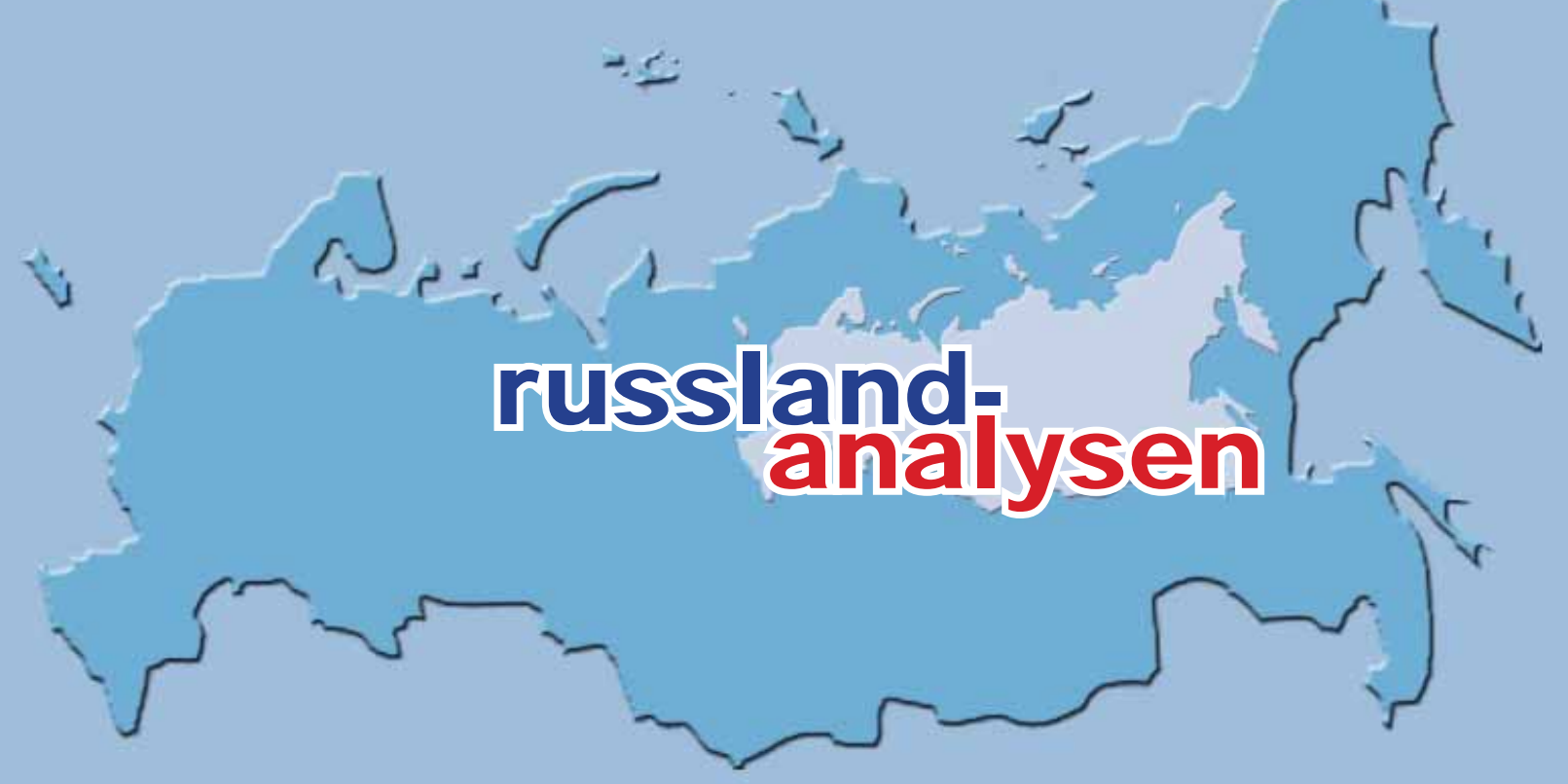

\title{
http://www.laender-analysen.de/russland/
}

\section{VON PUTIN ZU MEDWEDJEW}

\section{KOMMENTAR}

Chance für einen neuen Anfang?

Hans-Henning Schröder, Bremen/Berlin

ANALYSE

Die Ära Putin. Eine Bilanz in Meinungsumfragen

Hans-Henning Schröder, Bremen/Berlin

TABELLEN UND GRAFIKEN ZUM TEXT

Wie bewerten Sie heute die Arbeit Putins im Amt des Präsidenten?

Wie war das vergangene Jahr?

Was hat sich geändert: Die Jahre 2000-2007 in der Bewertung der Bevölkerung

DOKUMENTATION

Die vorläufigen Ergebnisse der Präsidentenwahlen vom 2. März 2008

UMFRAGE

Braucht Russland internationale Wahlbeobachter?

UMFRAGE

Die öffentliche Meinung zu Frauenrechten im internationalen Vergleich

CHRONIK

Vom 29. Februar bis zum 6. März 2008

\section{Forschungsstelle Osteuropa}

Deutsche Gesellschaft für Osteuropakunde
Otto Wolff Stiftung 


\section{Kommentar}

\section{Chance für einen neuen Anfang?}

Hans-Henning Schröder, Bremen/Berlin

\section{Die Technik der Machtübergabe}

Am 2. März wurde Dmitrij Medwedjew erwartungsgemäß mit überwältigender Mehrheit zum Nachfolger Wladimir Putins gewählt. Zum zweiten Mal ist es den Eliten des postsowjetischen Russland gelungen, für die translatio imperii-die Übergabe der obersten Macht an einen Nachfolger - die Zustimmung der Bevölkerung zu gewinnen. Wie bereits 1999/2000 erprobt, erfolgte die Auswahl und Bestätigung des Nachfolgers in mehreren Schritten: Präsident und Präsidialadministration wählen einen vertrauenswürdigen Nachfolger aus, der die Sicherheit des amtierenden Präsidenten und seiner Umgebung auch nach der Abgabe der Macht garantiert; der potentielle Nachfolger muss dann von den Eliten akzeptiert werden - der Elitenkonsens ist die Basis, die den Nachfolger trägt; und schließlich bedarf es eines Aktes plebiszitärer Zustimmung, der ihm eine formale Legitimation verschafft. Die Präsidentenwahl am 2. März war der dritte und letzte Akt dieses Übergabeprozesses. Weder 1999/2000 noch 2007/2008 verlief dieser Prozess reibungslos. 1999 schwankte die "Jelzin-Familie« zwischen wenigstens zwei Kandidaten und Putin war nur die zweite Wahl, nachdem sich erwies, dass Stepaschin keinen Rückhalt gewinnen konnte. Auch die Putin-Administration agierte im Herbst 2007 ausgesprochen zögerlich und präsentierte den Kandidaten Medwedjew erst, als die Nachfolgedebatte in Moskau bei vielen Beobachtern Irritationen ausgelöst hatte. Nach der öffentlichen Bestimmung des Kandidaten verlief der weitere Prozess aber bemerkenswert glatt und das Wahlvolk akklamierte den Nachfolger, der im Tandem mit seinem Vorgänger Putin antrat, in überzeugender Weise.

\section{Wandel der Machtverhältnisse?}

Der reibungslose Verlauf der Übergabeprozedur bedeutet allerdings nicht, dass die Machtverhältnisse nun schon konsolidiert sind. Die Mechanismen der neuen Doppelspitze bedürfen erst der Erprobung und die tatsächlichen Kräfteverhältnisse sind noch keineswegs geklärt. Auch die Putin-Administration hat Zeit gebraucht, um ihre Position zu konsolidieren. Putin selbst verfügte $1999-2000$ praktisch über keine eigene Hausmacht und hatte in der Gesellschaft keinen politischen Rückhalt. Dennoch konnte er allmählich eine Umgestaltung der Machtverhältnisse in die Wege leiten, die er mit einem Elitenwechsel begleitete. Putin nützte die Heterogenität der Elitenkoalition, die ihn trug, klug aus und eliminierte Schritt für Schritt die Gruppierungen, die ihn einengten. Er trieb jene Finanzmagnaten, die die Medien kontrollierten wie Wladimir Gusinskij und Boris Beresowskij, außer Landes, schwächte durch eine Reihe administrativer Maßnahmen die Position der Gouverneure und schuf sich eine Hausmacht, indem er Angehörige des FSB und seiner Petersburger Entourage in Schlüsselpositionen brachte. 2003 war die Putin-Administration stark genug, den reichsten Mann Russlands in Lagerhaft zu nehmen, sein Finanzimperium zu zerschlagen und die Teilstücke an die eigenen Parteigänger zu verteilen. Im Gefolge dieser Aktion wurden dann auch die letzten Vertreter des Jelzinschen Apparates aus dem Machtzentrum entfernt und mit der Abschaffung der Wahl der Gouverneure, deren Abhängigkeit vom Präsidenten gesichert.

Medwedjew wird es in mancher Hinsicht schwerer haben als Putin, da sein Vorgänger sich nicht aus der Öffentlichkeit zurückzieht. Jelzin war physisch außerstande, noch eine politische Rolle wahrzunehmen, Putin ist im besten Politikeralter und genießt hohes Ansehen bei Eliten und Bevölkerung. Allein aufgrund dessen stellt er einen politischen Faktor dar. Jelzin war nach seinem Rückzug nicht mehr als eine unangenehme Erinnerung. Medwedjew muss also mit Putin rechnen, doch er braucht auch die Rückendeckung, wenigstens solange, bis er eine eigene Statur gewonnen hat.

\section{Medwedjews »Neue Gesellschaft«}

Programmatisch kann Medwedjew an seinen Vorgänger anknüpfen. Putin hat die zahlreichen Probleme, mit denen das Land konfrontiert ist, immer wieder offen angesprochen - die fehlende Rechtstaatlichkeit, die Korruption, die Habgier der Beamten, die großen sozialen Unterschiede, die Missstände im Renten- und Gesundheitssystem, die Schwächen der Infrastruktur, die Mängel der verarbeitenden Industrie, die hinter der allgemeinen Entwicklung zurückgeblieben ist. Auf manchen Feldern besserte sich die Lage in den Putin-Jahren. So stiegen die Reallöhne und die Armut ging zurück, doch viele Probleme blieben ungelöst. So liegt die durchschnittliche Lebenserwartung 2006 für Männer bei 60 und für Frauen bei 73 Jahren - für ein 


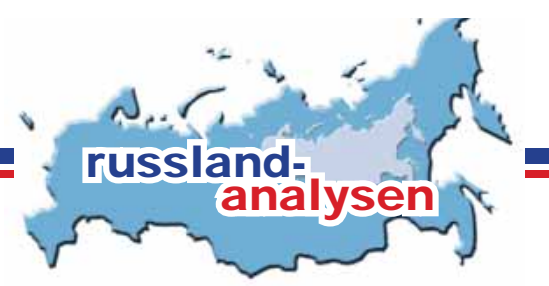

Industrieland ein beschämend niedriger Wert. Hier liegen die Aufgaben, die Medwedjew lösen muss.

Und ganz richtig hat er sie in den programmatischen Reden angesprochen, die er im Januar und Februar 2008 in verschiedenen Kontexten gehalten hat. In Krasnojarsk entwarf er am 15. Februar eine Vision von persönlicher Freiheit in einem funktionierenden Rechtsstaat. Er kritisierte die »Geld-« und die »Telefonrechtsprechung « - die gängige Praxis, Gerichtsentscheidungen durch Bestechung oder Anrufe smächtiger Freunde zu beeinflussen. Medwedjew verlangte auch die Beseitigung der Korruption und eine durchgreifende Verbesserung der Verwaltung bei gleichzeitigem Personalabbau, u.a. um die Entwicklung der mittelständischen Wirtschaft zu fördern. Respekt vor dem Eigentum und vor Rechtsvorschriften stellte er als Kern einer erfolgreichen Wirtschaftsentwicklung dar, zu der aber auch eine Entwicklung des Finanzsystems und der Verkehrs- und Kommunikationsinfrastruktur gehöre. Medwedjew verlangte mehr technische und wissenschaftliche Innovation, eine bessere Wissenschaftspolitik und - als unabdingbare Voraussetzung - den Ausbau der sozialen Sphäre, um das Potential aller Menschen zu wecken, und eine »neue Gesellschaft« zu schaffen, in der jeder "motiviert ist, Waren und Dienstleistungen höchster Qualität zu produzieren und zu konsumieren".

Was Medwedjew in Krasnojarsk ankündigte, war eine Politik des »New Deal«, eine soziale Vision, die eine Perspektive für rasches Wachstum und eine allgemeine Besserung der Lebenssituation versprach. Vor dem Hintergrund hoher Energieeinnahmen erscheint dieses Programm auch nicht unrealistisch. Andererseits greift der Nachfolger alle jene Themen auf, die sein Vorgänger angesprochen hat, ohne sie wirklich einer Lösung zuzuführen. Medwedjews Programm erwächst aus der Benennung der Defizite der PutinJahre. Ob er aber willens und in der Lage ist, dieses Programm einzulösen, das muss vorläufig dahingestellt bleiben. Bei den so genannten »nationalen Projekten« in den Bereichen Landwirtschaft, Bildung, Gesundheitswesen und Wohnungsbau, die von Medwedjew koordiniert wurden, gab es bis jetzt kaum fühlbare Ergebnisse. Ob es dem Nachfolger gelingen wird, seine Macht - mit oder ohne Putin - zu konsolidieren, ob er sein Programm einer »neuen Gesellschaft« wirklich in Angriff nehmen will und ob er in der Lage ist, es gegen die starken Interessen des Verwaltungsapparats und der großen Finanzgruppen durchzusetzen, das muss sich in den nächsten Jahren erweisen.

Über den Autor

Prof. Dr. Hans-Henning Schröder lehrt osteuropäische Zeitgeschichte an der Universität Bremen.

\section{Lesetipps}

- Andrew Wilson: Meeting Medvedev: The Politics of the Putin Succession. Policy Brief. ECFR/05 February 2008 w w w.ecfr.eu

- Carl Holmberg: Managing Elections in Russia. Mechanisms and Problems. FOI Report (FOI-R--2474--SE), Stockholm : FOI 2008, 116 S. http:// w w w2 .foi.se/ rapp/ fo ir2 474 .pdf, 3. März 2008

- Marie Mendras: Le citoyen en danger. Droits de l'homme et libertés dans la Russie poutinienne. Février 2008 http:// w w w.euro pa rl.europa.eu/ a ctivities/ expert/ eS tud ies.d o? lang ua ge=FR, 12. Februar 2008

- Mark A Smith: The Russian Presidential Succession. Defence Academy of the United Kingdom Advanced Research and Assessment Group. Russian Series 08/01 http:// w w w.da.mod.uk/ colleges/ csrc/ document-listings/ russian/ $08 \% 2801 \% 29$ M AS.pdf, 17. Januar 2008 


\section{Analyse}

\section{Die Ära Putin. Eine Bilanz in Meinungsumfragen}

Hans-Henning Schröder, Bremen/Berlin

\section{Zusammenfassung}

Repräsentative Umfragen zu den Putin-Jahre zeigen, dass der russische Präsident in der Bevölkerung hohe Akzeptanz genießt. Gespeist wird diese positive Haltung zum Regime durch die fortschreitende Besserung der Lebensverhältnisse, die die russischen Bürger seit 1999 erfahren haben. Wirtschaftswachstum und Anstieg der Reallöhne werden mit dem Handeln des Staatsoberhaupts in Verbindung gebracht, das seit September 1999 eine führende Rolle in der Politik einnimmt.

Andererseits äußert die Bevölkerung am politischen System insgesamt und an seiner Funktionsweise berechtigte Kritik. Die ungleiche Vermögensverteilung, die Mängel des Gesundheitssystems und die perzipierten Sicherheitsprobleme nähren ein tiefes Misstrauen gegen den Staat und die politische Klasse, die ihn besitzt. Gewiss wird dieses Unbehagen gegenwärtig durch das Vertrauen zu Putin und die Besserung der Lebensverhältnisse überlagert, doch muss sich die Administration mittelfristig diesen Problemen stellen.

\section{Wahrnehmungen der Putin-Jahre}

Über Russland wussten die deutschen Medien in den letzten Jahren vorwiegend Negatives zu berichten: es war die Rede von einem autoritären Regime, der Unterdrückung von Oppositionellen, der Manipulation von Wahlen, von Aufrüstung und aggressivem Auftreten gegenüber dem Westen, man warnte vor Großunternehmern, die westliche Firmen aufkaufen - kurz die 'Gefahr aus dem Osten` war wieder präsent. Diese Warnungen hatten durchaus eine reale Basis. In der Tat hat die Putin-Administration im Innern politische Rechte eingeschränkt und nach außen die eigenen Interessen prononciert vertreten. Bei der westlichen Bewertung schwingen aber auch wieder russophobe Töne mit, die nicht unbedingt zu einer nüchternen Bewertung der russischen Entwicklung beitragen.

Vielleicht ist es dazu hilfreich, die Perspektive zu wechseln und zu prüfen, wie die Arbeit der PutinAdministration in Russland selbst perzipiert wird. Die russischen Bürger haben Augen und Ohren. Sie nehmen die alltäglichen Veränderungen in der Versorgung, in der Politik, in der Wirtschaft sehr viel deutlicher wahr, als man dies in Berlin, London oder Paris tun kann, und sie bewerten sie vor dem Hintergrund der Erfahrungen, die sie in der Sowjetzeit und in den Jelzin-Jahren gemacht haben. Will man also ein Urteil über die Putin-Administration fällen, lohnt es sich, die Vorstellungen der Regierten, der russischen Bevölkerung mit heranzuziehen.

Für ein solches Vorhaben stehen in großer Menge Umfragedaten zur Verfügung, die von verschiedenen russischen Instituten erhoben wurden, deren Arbeits- weise und Methodik entsprechenden Instituten in den USA oder Deutschlands durchaus vergleichbar ist.

\section{Aufschwung und hohe Zustimmungsraten}

Diese Erhebungen zeigen, dass Putin am Ende seiner zweiten Amtszeit in der Bevölkerung breite Unterstützung genießt. Nach einer Umfrage der "Stiftung Öffentliche Meinung (FOM) (vgl. Grafik S. 8 oben: Wie bewerten Sie heute die Arbeit Putins im Amt des Präsidenten? (Dezember 2007)) bewerten etwa zwei Drittel der Befragten die Tätigkeit Putins als Präsident mit "gut" oder "ausgezeichnet«. Diese Zustimmung ist weitgehend unabhängig vom Geschlecht, dem Bildungs- oder Eigentumsniveau und dem Wohnort. Der scheidende Präsident verfügt über Rückhalt in allen Schichten der Bevölkerung, und damit auch über die Legitimation, für ganz Russland zu sprechen.

Der Hauptgrund für die hohen Zustimmungsraten ist zweifellos die fortgesetzte Besserung der Lebensverhältnisse, die die russische Bevölkerung in der Regierungszeit Putins erfahren hat. Das Lewada-Zentrum, eines der führenden russischen Meinungsforschungsinstitute, fragt jedes Jahr im Dezember danach, wie das vergangene Jahr im Vergleich zum Jahr zuvor bewertet wird. In den neunziger Jahren - unter Jelzin - gaben die Befragten in der Regel an, das gerade ablaufende Jahr sei schwerer gewesen als das vorher. Eine Wende zeichnete sich $1997 \mathrm{ab}$ - es schien fast, man sei rüber den Berg $<-$, doch dann folgte das schreckliche Jahr 1998 mit der schweren Finanzkrise im Herbst und dem Einbruch des Rubelkurses. 1999 begann sich die Situation nachhaltig zu bessern und die Bevölkerung 
brachte diese Erholung mit Putin in Verbindung, der im September 1999 Ministerpräsident geworden war, Ende Dezember 1999 nach dem Rücktritt Jelzins in das Amt des Präsidenten einrückte, um dann im März 2000 auch regulär gewählt zu werden.

Wachsende Zufriedenheit mit den Lebensverhältnissen ist die Basis für die hohen Zustimmungsraten zu Präsident Putin - Zustimmungsraten, die sich auch in Wahlerfolge ummünzen lassen, wie das zuletzt bei den Parlamentswahlen im Dezember 2007 und jetzt bei den Präsidentenwahlen 2008 geschehen ist. Das Vertrauen in den Präsidenten verleiht dem politischen System auch eine hohe Stabilität - insofern und solange Putin in den Augen der Bevölkerung eine maßgebliche politische Rolle spielt. Daher ist es ganz folgerichtig, dass die Führung auch nach dem März 2008, wenn ein Personalwechsel im Präsidentenamt erfolgt, eine hohe Position für Putin ins Auge fasst - sei es nun das Amt des Ministerpräsidenten oder ein anderes.

\section{Wahrnehmungen der Putin-Ära}

Die Untersuchungen des Lewada-Zentrums erlauben es, ein schärferes Bild von der Gemütslage der Bevölkerung zu zeichnen. Das Umfrageinstitut hat die Stimmung der russischen Bevölkerung über all die PutinJahre hinweg verfolgt und die Daten Ende 2007 veröffentlicht. Aus den repräsentativen Erhebungen ergibt sich ein differenziertes Bild. Erfragt wurden Perzeptionen - Wahrnehmungen einer Entwicklung, nicht die Entwicklung selbst. Aber diese sind von erheblicher politischer Bedeutung, denn es sind die Perzeptionen, die politische Haltungen bestimmen. Die Analyse der Perzeptionsentwicklung kann also Auskunft geben über Hintergründe der hohen Zustimmungsraten für die Putin-Administration. Sie kann aber auch mögliche Konfliktpunkte und latente Spannungen aufzeigen, auf die die Politik in der nächsten Amtszeit reagieren muss.

Die erste Beobachtung kommt nicht überraschend - sie ergibt sich schon aus der oben zitierten Umfrage (vgl. Grafik S. 8 unten: Wie war das vergangene Jahr für Russland: schwerer als das vorhergehende Jahr, leichter oder genauso?) -, nämlich, dass die wirtschaftliche Erholung eine Konstante der Putin-Jahre war, die sich auch in einer allgemeinen Besserung der materiellen Verhältnisse niederschlägt. Die überwiegende Mehrheit der russischen Bevölkerung registrierte, dass sich das Angebot an Lebensmitteln (vgl. Grafik S. 9 unten: Wie hat sich das Angebot von Lebensmitteln geändert?), an Kleidung und Artikeln des täglichen Grundbedarfs von Jahr zu Jahr besserte. Besonders in der ersten Amtszeit Putins 2000-2004, als die Gesellschaft noch unter dem Eindruck der schwierigen Jel-
zin-Jahre und der Finanzkrise des Herbstes 1998 stand, empfand man, dass es unter dem neuen Präsidenten aufwärts ging. In der zweiten Amtszeit wirkte die Verbesserung des Konsumangebots offenbar schon wie selbstverständlich. Erst im Wahljahr 2007 stieg der Anteil derjenigen, die eine Besserung gegenüber dem Vorjahr sahen, noch einmal auf $66 \%$ - zwei Drittel der Bevölkerung.

Fragt man nach den individuellen Einkommenschancen (vgl. Grafik S. 12 unten: Wie haben sich die Möglichkeiten verändert, gut zu verdienen?), nicht nach dem allgemeinen Konsumniveau, kann man zwar einen ähnlichen Trend beobachten - Anstieg des Anteils der Befragten mit verbesserten Einkommenserwartungen in den Jahren 2000-2002 und 2007, leichter Rückgang und Stagnation 2003-2006 -, doch überwiegt bis 2005 der Anteil derjenigen, die eine Verschlechterung der Einkommenssituation wahrnehmen. Eine wirkliche Wende ist erst 2007 erkennbar. Für die Mehrheit der Befragten wurde die Besserung der eigenen materiellen Lage also nur verzögert fühlbar. Die positive Wahrnehmung der Präsidentschaft Putins und der Lage des Landes bestätigte sich mit einer gewissen Verspätung nun auch im Bereich des individuellen Lebens.

\section{Sozialpolitische Problemfelder}

Die allgemein positive Bewertung der Situation verstärkt zweifellos die Akzeptanz, die Putin und seine Administration genießen. Politisch führt dies zu einer Stabilisierung - jedenfalls so lange Putin der Führung angehört. Allerdings lassen sich anhand der Umfragen auch eine Reihe von Problemen identifizieren, mit denen sich die russische Regierung in den nächsten Jahren auseinandersetzen muss.

Dazu gehört vor allem der verbreitete Eindruck, dass die russische Gesellschaft nicht gerecht organisiert ist. Bei der Frage nach Verteilungsgerechtigkeit (vgl. Grafik S. 14 unten: Wie hat sich die Gerechtigkeit bei der Verteilung materieller Güter geändert?) gibt es kaum Respondenten, die positiv antworten. Zwischen 40 und $50 \%$ sehen eine Vergrößerung der sozialen Unterschiede, während der Anteil derjenigen, die eine Verbesserung erkennen wollen, die ganze Amtszeit Putins über im einstelligen Bereich liegt. Offenbar ist es der Putin-Administration trotz des Wirtschaftswachstums und der allgemeinen Besserung der materiellen Verhältnisse nicht gelungen, den Gegensatz zwischen Arm und Reich, der mit der Privatisierung und dem Übergang zum Markt in den neunziger Jahre entstanden ist, zu mildern oder wenigstens den Eindruck zu vermitteln, man strebe dies an.

Die tiefe Spaltung der Gesellschaft, die von breiten Kreisen der Bevölkerung wahrgenommen wird, 
stellt für jede Administration eine Belastung dar. Die latente soziale Spannung, die in den Putin-Jahren durch die Verbesserung der materiellen Verhältnisse überlagert wurde, könnte - wenn die Wirtschaftsentwicklung nicht mehr positiv verläuft - auch politisch Bedeutung gewinnen. Schon jetzt profitiert von ihr die Kommunistische Partei, die sich trotz aller Restriktionen hartnäckig im Parlament hält. Auch der Wahlerfolg der Partei "Heimat" (Rodina) im Jahre 2004 war damit verbunden, dass diese Partei neben der nationalistischen auch die soziale Karte spielte.

Soziale Ungleichheit, das Gefühl, dass es in der Gesellschaft ungerecht zugeht, ist eines der Probleme, das auf das politische Klima einwirkt. Zwei weitere Fragen stehen damit in Zusammenhang - die Gesundheitsversorgung und die persönliche Sicherheit. In beiden Fällen ist es der Putin-Administration offenbar nicht gelungen, fühlbare Verbesserungen durchzusetzen. Das Gesundheitswesen wird in der Bevölkerung nach wie vor als Problemsektor wahrgenommen (vgl. Grafik S. 10 unten: Wie hat sich die Arbeit der Krankenhäuser, Polikliniken geändert?). Noch 2007 wagen kaum 16 \% von Verbesserungen in diesem Bereich zu sprechen. Dieser Anteil wird weit übertroffen von der Zahl derjenigen, die im siebten Jahr der Putinschen Präsidentschaft davon ausgehen, dass sich die Situation in Krankenhäusern und Polikliniken weiter verschlechtert hat. Diese Perzeption stellt der Gesundheitspolitik der Putin-Administration ein denkbar schlechtes Zeugnis aus.

Ähnlich stellt sich die Situation bei der Wahrnehmung der persönlichen Sicherheit dar (vgl. Grafik S. 13 unten: Wie hat sich die Sicherheitslage der Bürger geändert?). Gewiss ist der Anteil der Bürger, die eine Verschlechterung der Sicherheitssituation erkennen wollen, zwischen 2004 und 2006 zurückgegangen, doch interessanterweise schnellt ihr Anteil 2007 von $33 \%$ auf $44 \%$ hoch. In diesen Schwankungen wird deutlich, dass trotz Konsolidierung der materiellen Situation das Misstrauen in die Fähigkeit des Staates, für die Sicherheit des einzelnen zu sorgen, in erheblichem Maße fortbesteht. Hier manifestiert sich eine schwer zu überwindende Skepsis gegenüber Polizei und Gerichten.

Das Gefühl sozialer Ungerechtigkeit und die Vorstellung, dass der Staat weder für die Gesundheit noch für die Sicherheit der Bürger ausreichend sorgt, scheint in beiden Amtszeiten Putins für die Haltung der Mehrzahl der Bürger gegenüber dem System und den Behörden bezeichnend zu sein. Doch hat das offensichtliche Unbehagen keine unmittelbaren Folgen für die Einstellung gegenüber der Person des Präsidenten. In einer Phase, die durch wirtschaftliches Wachstum und allmähliche Erholung der materiel- len Lage bestimmt ist, werden diese Wahrnehmungen von der Erwartung einer fortgesetzten Besserung überlagert. Langfristig belasten sie dennoch das Verhältnis zwischen Staat und Gesellschaft und eine künftige Administration ist gut beraten, eine Politik sozialen Ausgleichs zu betreiben, das Rechtssystem zu stabilisieren und eine Gesundheits- und Sozialreform durchzusetzen.

\section{Meinungsfreiheit und politische Partizipation}

Geht man von den Umfragedaten aus, so gibt es eine Mehrheit in Russland, die über die Entwicklung der Sozialordnung, des Gesundheits- und des Rechtsschutzbereichs verdrossen ist. Bei der Bewertung des politischen Systems hingegen ist der Anteil der Kritiker nicht ganz so hoch. Während die westliche Öffentlichkeit eine rapide Einengung der Freiheitsrechte in Russland unterstellt, nimmt die russische Öffentlichkeit keine dramatische Verschlechterung der politischen Verhältnisse wahr.

Lediglich 15-20\% der Befragten sehen einen Niedergang der Meinungsfreiheit. Die Mehrheit erkennt keine Veränderungen, ja 2007 will mehr als ein Drittel der Befragten eine Verbesserung in diesem Bereich feststellen (vgl. Grafik S. 14 oben: Wie hat sich die Möglichkeit verändert, seine Meinung frei zu äußern?). Das mag damit zusammenhängen, dass manche noch die Situation der Sowjetzeit vor Augen haben, von der sich die Lage der Jahre 2000-2007 trotz mancher Einschränkungen immer noch dramatisch positiv unterscheidet. Zum anderen muss man sich vor Augen halten, dass das Fernsehen zwar weitgehend von der Putin-Administration kontrolliert wird, Presse und Buchhandel aber vielfach noch frei agieren. So sind z.B. kritische Werke von Anna Politkowskaja oder eine Interviewsammlung des emigrierten >Oligarchen`Boris Beresowskij in Moskau frei verkäuflich. Und Zeitungen wie »Vedomosti« oder »Kommersant» berichten über viele Politikbereiche durchaus kritisch. Die Mehrheit der russischen Bürger erfährt daher die Putin-Jahre nicht unbedingt als Phase, in der die Meinungsfreiheit eingeschränkt wurde.

Allerdings sind die Befragten - realistischerweise - nicht sonderlich optimistisch, wenn es um ihre persönliche Möglichkeiten geht, auf Staatsgeschäfte Einfluss zu nehmen (vgl. Grafik S. 13 oben: Wie hat sich die Möglichkeit einfacher Menschen verändert, auf staatliche Angelegenheiten Einfluss zu nehmen?). Das Gefühl "Die da oben machen sowieso, was sie wollen « ist auch in westlichen Gesellschaften nicht unbekannt, und in Putins Russland mit seinen Unterschieden zwischen Arm und Reich und seinem Herrschaftsapparat, der gemeinhin nur "die Macht" (wlast) genannt wird, 
weit verbreitet. Dieses Gefühl hat sich im Verlauf der Amtszeit Putins nicht wesentlich verändert. Kennzeichnend (und selbsterklärend) ist lediglich, dass in den Wahljahren 2003 und 2007 der Anteil derjenigen geringfügig zunimmt, die die Ansicht vertreten, die Bürger könnten, auf Politik Einfluss zu nehmen.

\section{Fazit}

Die Umfragen aus den Putin-Jahre zeigen, dass der russische Präsident in der Bevölkerung hohe Akzeptanz genießt. Die plebiszitäre Zustimmung ist zweifellos ein wichtiger Aspekt der Machtstellung, die er innehat. Gespeist wird diese positive Haltung zum Regime durch die fortschreitende Besserung der Lebensverhältnisse, die die russischen Bürger seit 1999 erfahren. Das Wirtschaftswachstum und das Ansteigen der Reallöhne wird mit dem Handeln des Staatsoberhaupts in Verbindung gebracht, das seit September 1999 eine führende Rolle in der Politik einnimmt. Die Identifikation der Bürger mit Putin ist ein stabilisierendes Element für das politische System. Das macht verständlich, warum die politische Klasse nach Möglichkeiten sucht, Putin auch nach dem Präsidentenwechsel im März 2008 in den Führungsapparat einzubauen.

Dies ist auch deshalb notwendig, weil die Bevölkerung am politischen System insgesamt und an seiner Funktionsweise berechtigte Kritik hat. Die ungleiche Vermögensverteilung, die Mängel des Gesundheitssystems und die perzipierten Sicherheitsprobleme nähren ein tiefes Misstrauen gegen den Staat und die politische Klasse, die ihn besitzt. Gewiss wird dieses Unbehagen gegenwärtig durch das Vertrauen zu Putin und die Besserung der Lebensverhältnisse überlagert, doch muss sich die Administration mittelfristig diesen Problemen stellen. Dmitrij Medwedjew, der neue Präsident muss alles daran setzen, das Vertrauen der Gesellschaft zu gewinnen. Ein Schritt in diese Richtung wäre es, wenn die so genannten »nationalen Projekte«, mit denen im Bereich der Gesundheits- und Bildungspolitik, im Wohnungsbau und in der Landwirtschaft eine Besserung der Verhältnisse durchgesetzt werden soll, mit größerer Energie weiterverfolgt würden als bisher. Die Ausweitung dieser Maßnahmen zu einer regelrechten Politik des »New Deal«, die auf soziale Chancengleichheit setzt, würde die Position der neuen Administration sicher stärken und mittelfristig die Gefahr mindern, dass die vorhandenen sozialen Konflikte aufbrechen.

Meinungsfreiheit und politische Partizipation indes sind keine Themen, die die Bevölkerung beunruhigen. Die Entwicklung in den Medien empfindet eine Mehrheit durchaus als positiv. dass die Möglichkeiten zur politischen Beteiligung eingeschränkt sind, nimmt die Gesellschaft wahr, ohne dass dies ihre Zustimmung für den Präsidenten beeinträchtigt.

Europäische Politik findet Anknüpfungspunkte am ehesten im Bereich der Sozial- und Gesundheitspolitik. Will die Medwedjew-Administration wirklich die Strukturprobleme Russlands angehen, so ist eine Zusammenarbeit im Sozial- und Gesundheitsbereich denkbar. Beim Wohnungsbau etwa verfügt die Bundesrepublik über reiche Erfahrung und erfolgreiche Modelle. Im Bildungssystem hat die Einbindung Russlands in den Bologna-Prozess schon begonnen und kann vertieft werden. Hier bietet sich eine Vielzahl von Möglichkeiten zu Austausch und zur engen Zusammenarbeit.

\section{Über den Autor}

Prof. Dr. Hans-Henning Schröder lehrt osteuropäische Zeitgeschichte an der Universität Bremen.

\section{Lesetipps}

- Lev Gudkov: Staat ohne Gesellschaft. Zur autoritären Herrschaftstechnologie in Russland, in: Osteuropa, 58.2008, Nr. 1, S. 3-16.

- Boris Dubin: Gesellschaft der Angepassten. Die Brežnev-Ära und ihre Aktualität, in: Osteuropa, 57.2007, Heft 12, S. 65-78.

- Abbott, Pamela; Wallace, Claire: Talking About Health and Well-Being in Post-Soviet Ukraine and Russia, in: Journal of Communist Studies and Transition Politics, 23.2007, Nr. 2, S. 181-202.

- Vladimir Shlapentokh: Trust in public institutions in Russia: The lowest in the world, in: Communist and PostCommunist Studies, 39-2006, No. 2, S. 153-174. 


\section{Tabellen und Grafiken zum Text}

\section{Wie bewerten Sie heute die Arbeit Putins im Amt des Präsidenten?}

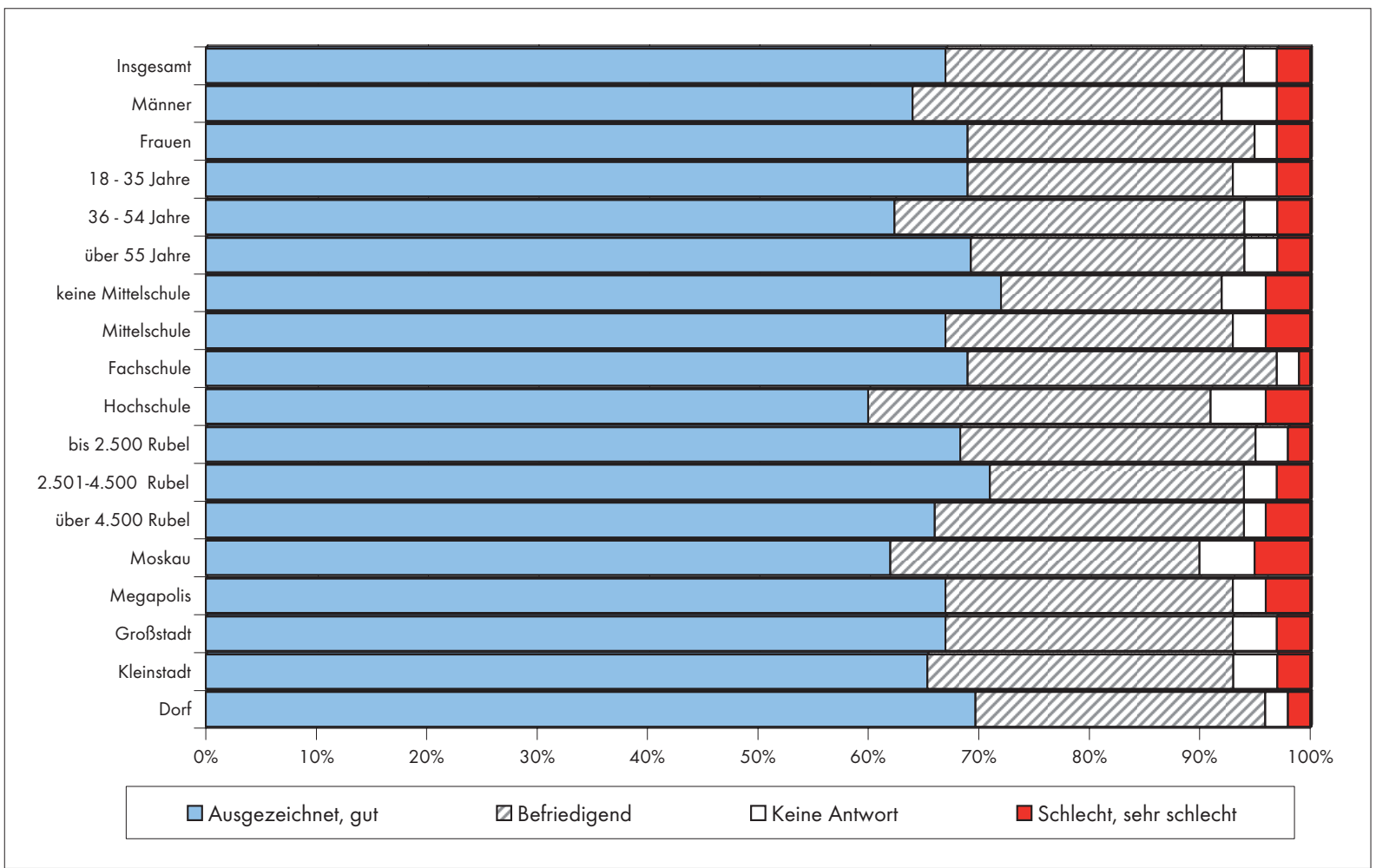

Nach: http :// bd.fo m.ru/ report/ map/ projects/ do minant/ d om0752/ d 075202 \#Abs1, 27. Dezember 2007

\section{Wie war das vergangene Jahr?}

Wie war das vergangene Jahr für Russland?

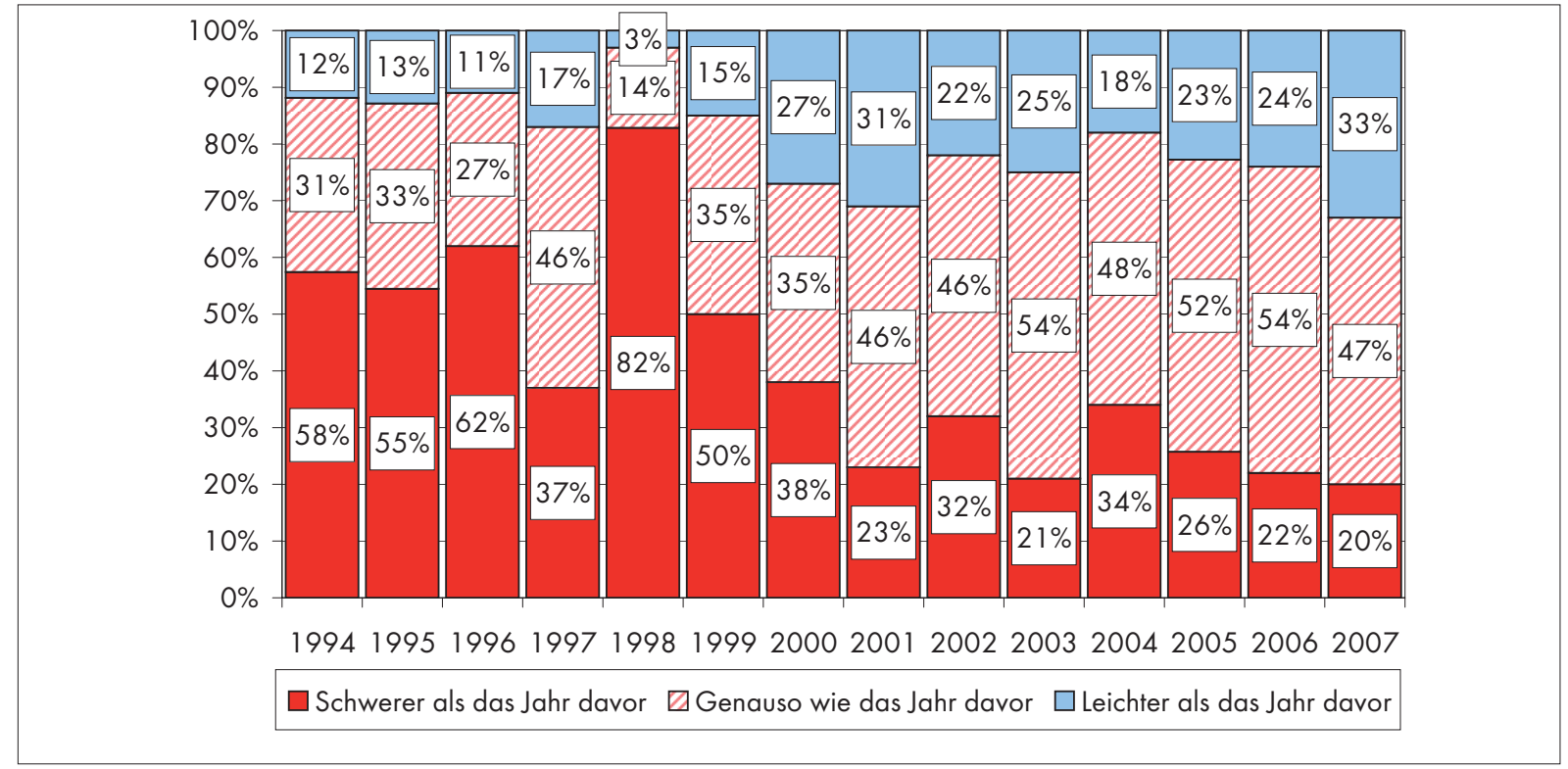

Quelle: Jährliche Umfragen des Lewada-Zentrums, zuletzt erhoben im Dezember 2007 http:// w w w.leva da.ru/ ta b l13.html 
Wie war das vergangene Jahr für Sie und Ihre Familie?

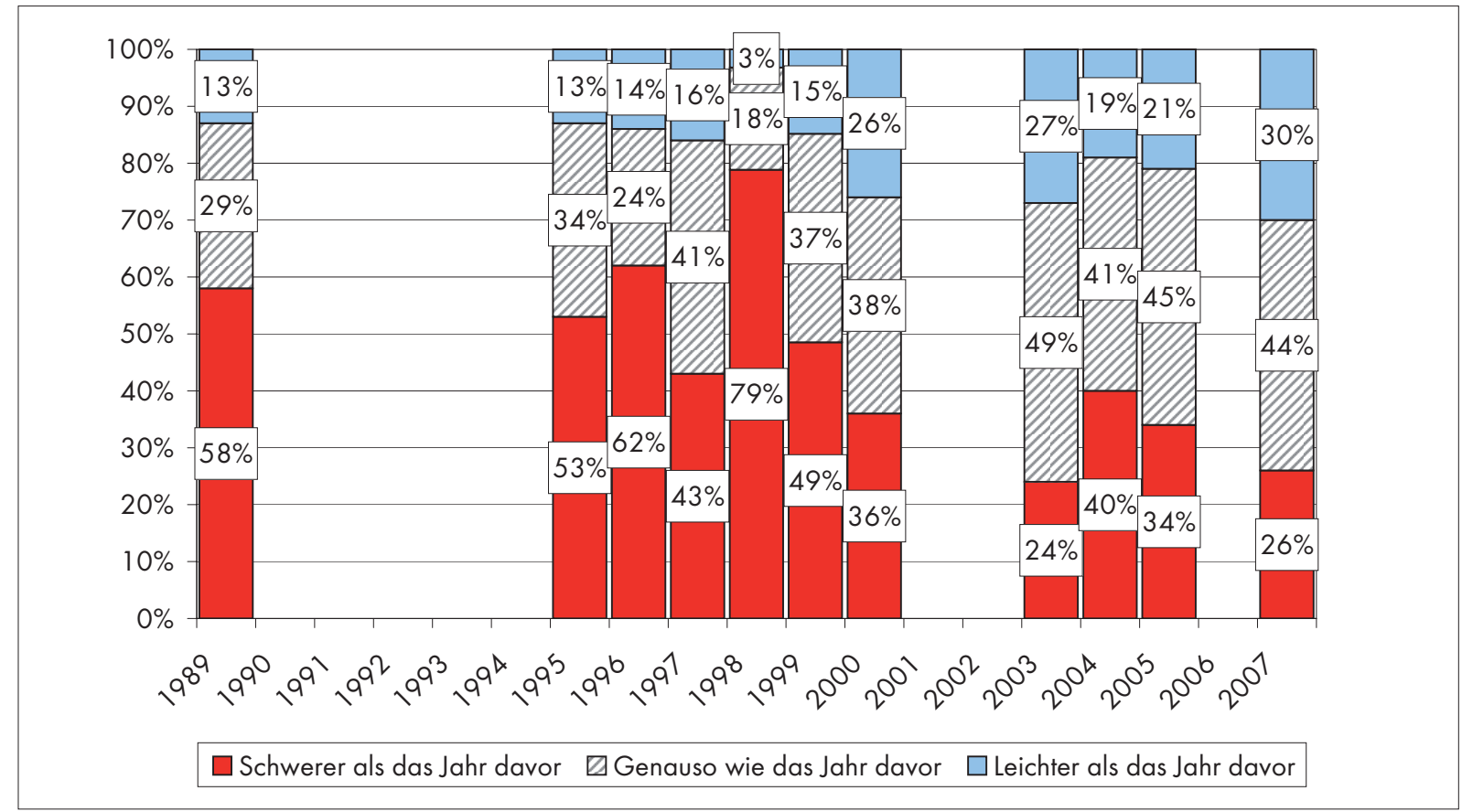

Quelle: Jährliche Umfragen des Lewada-Zentrums, zuletzt erhoben im Dezember 2007 http:// w w w.leva da.ru/ ta b l13.html

\section{Was hat sich geändert: Die Jahre 2000-2007 in der Bewertung der Bevölkerung}

Eine deutsche Übersetzung der Lewada-Daten findet sich unter:

http:// w w w.russlandanalysen.de/ content/ media/ Russlandanalysen15 4 .pdf

Veränderungen beim Angebot von Lebensmitteln?

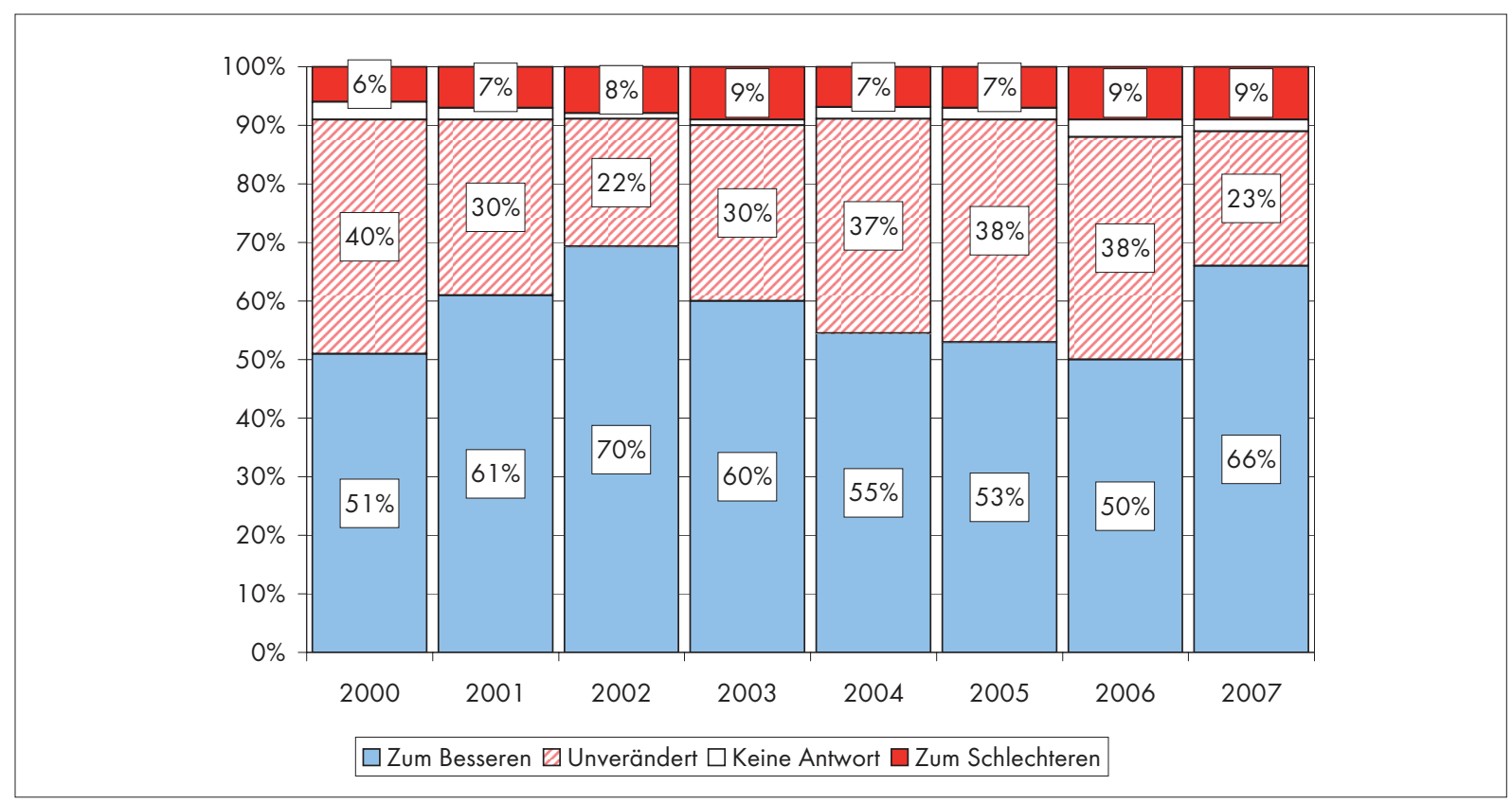

Quelle: Umfragen des Lewada-Zentrums, 20.-23. November 2007 http :// w w w.leva da .ru./ press/ 2007120703. html 
Veränderungen beim Angebot von Kleidung, Schuhen, Grundbedarfsartikeln?

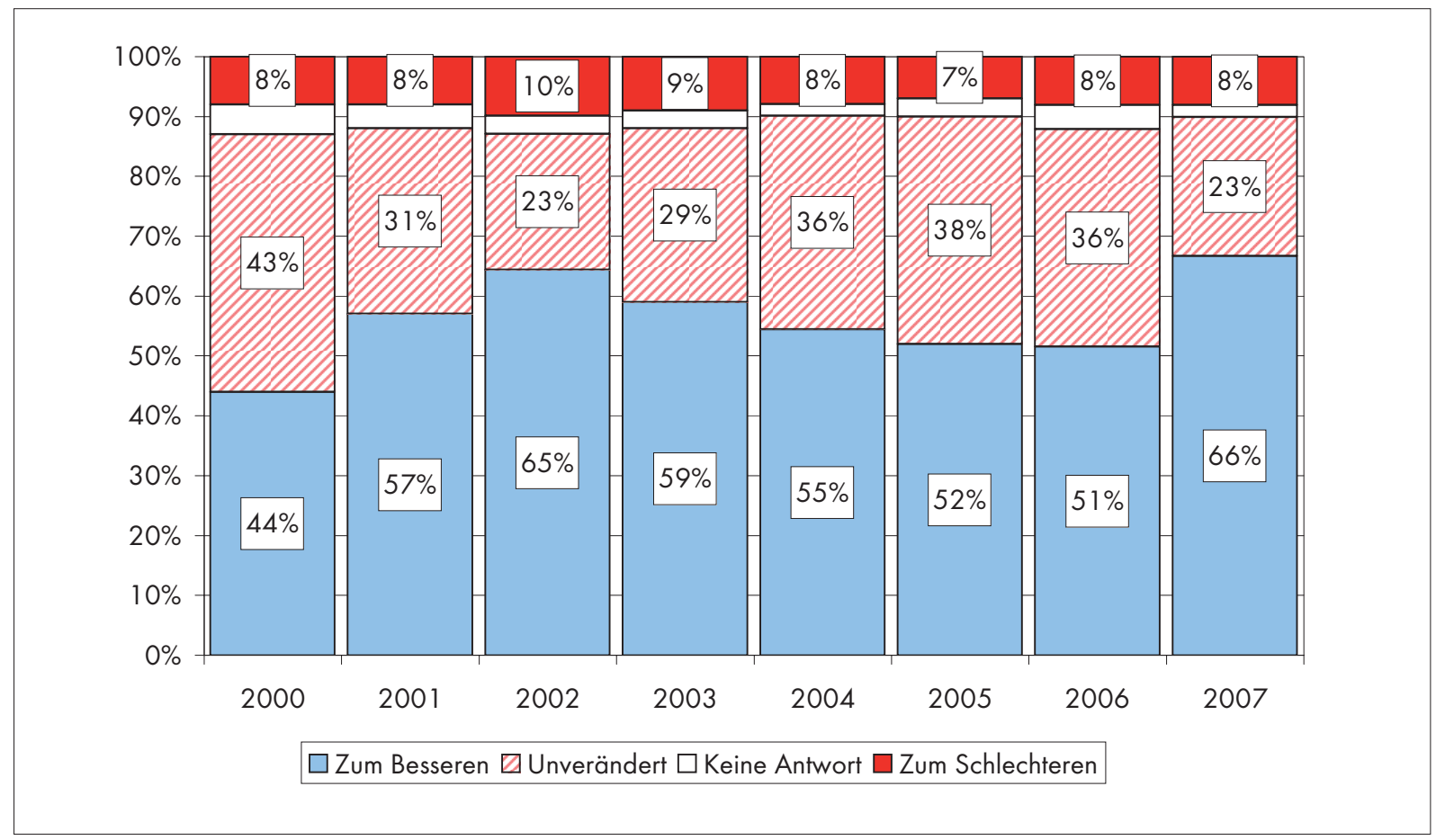

Veränderungen in der Arbeit der Krankenhäuser, Polikliniken?

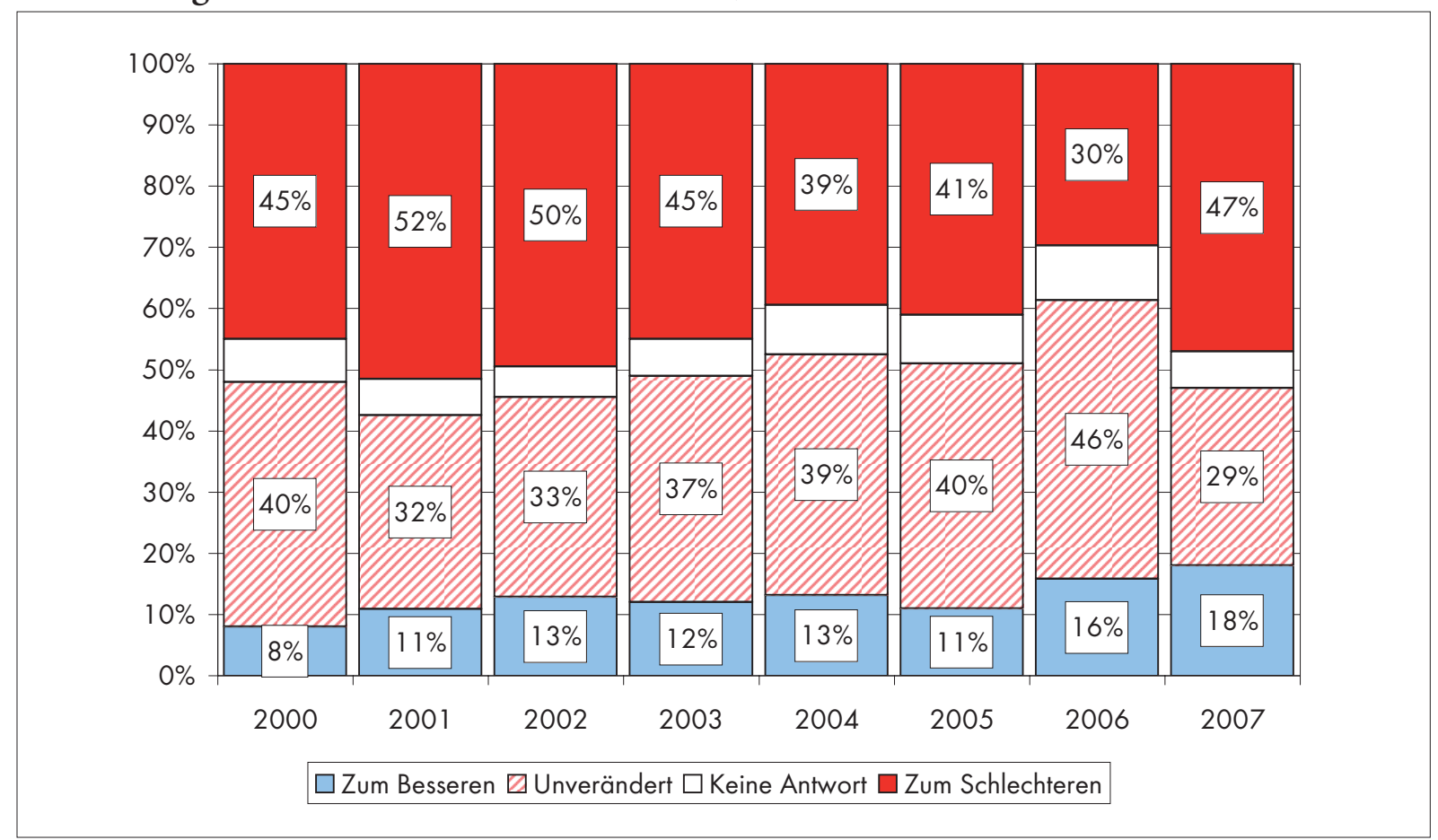


Veränderungen bei der Arbeit von Miliz und Rechtsschutzorganen?

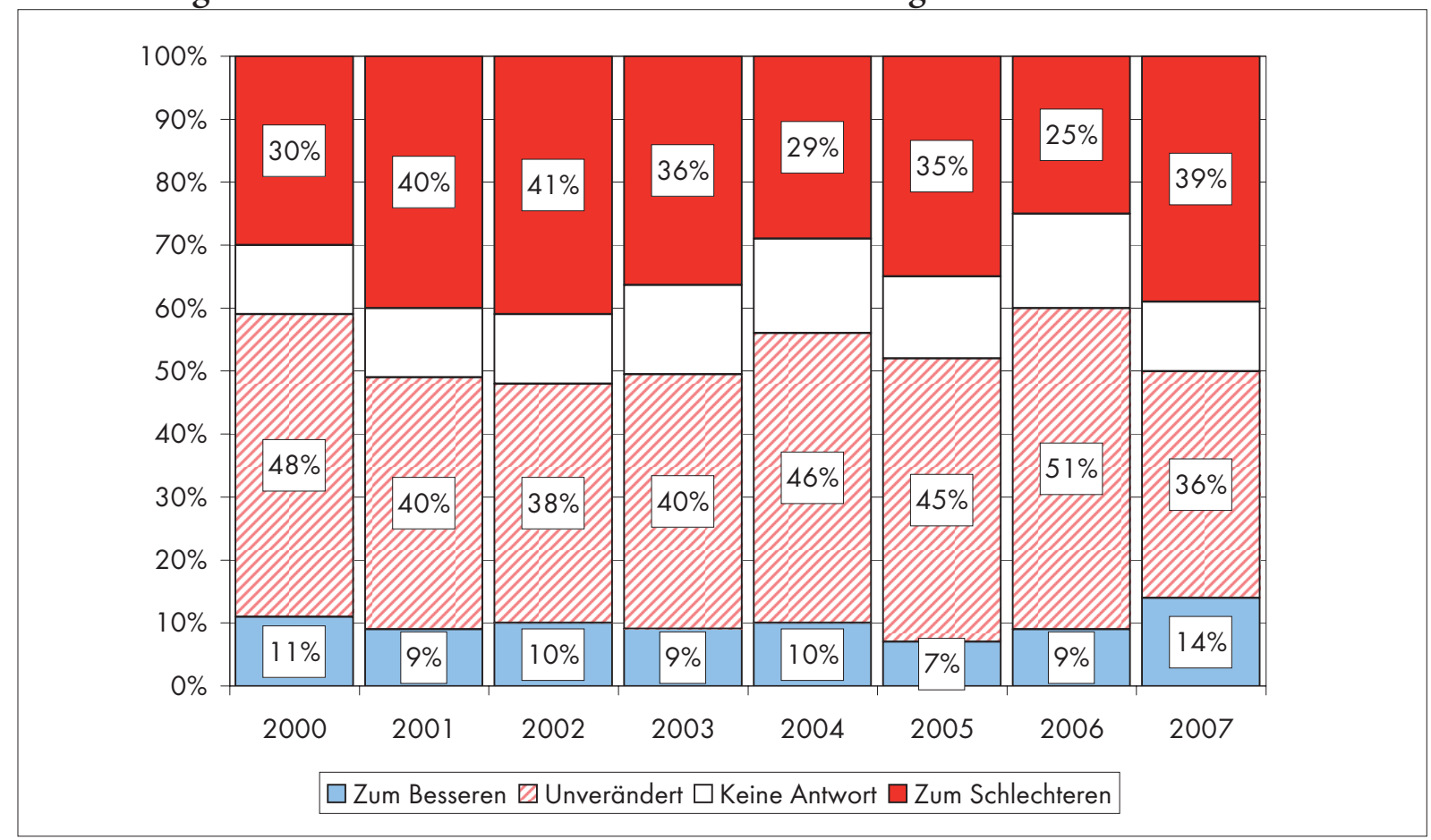

Veränderungen in der Tätigkeit von Presse, Radio und Fernsehen?

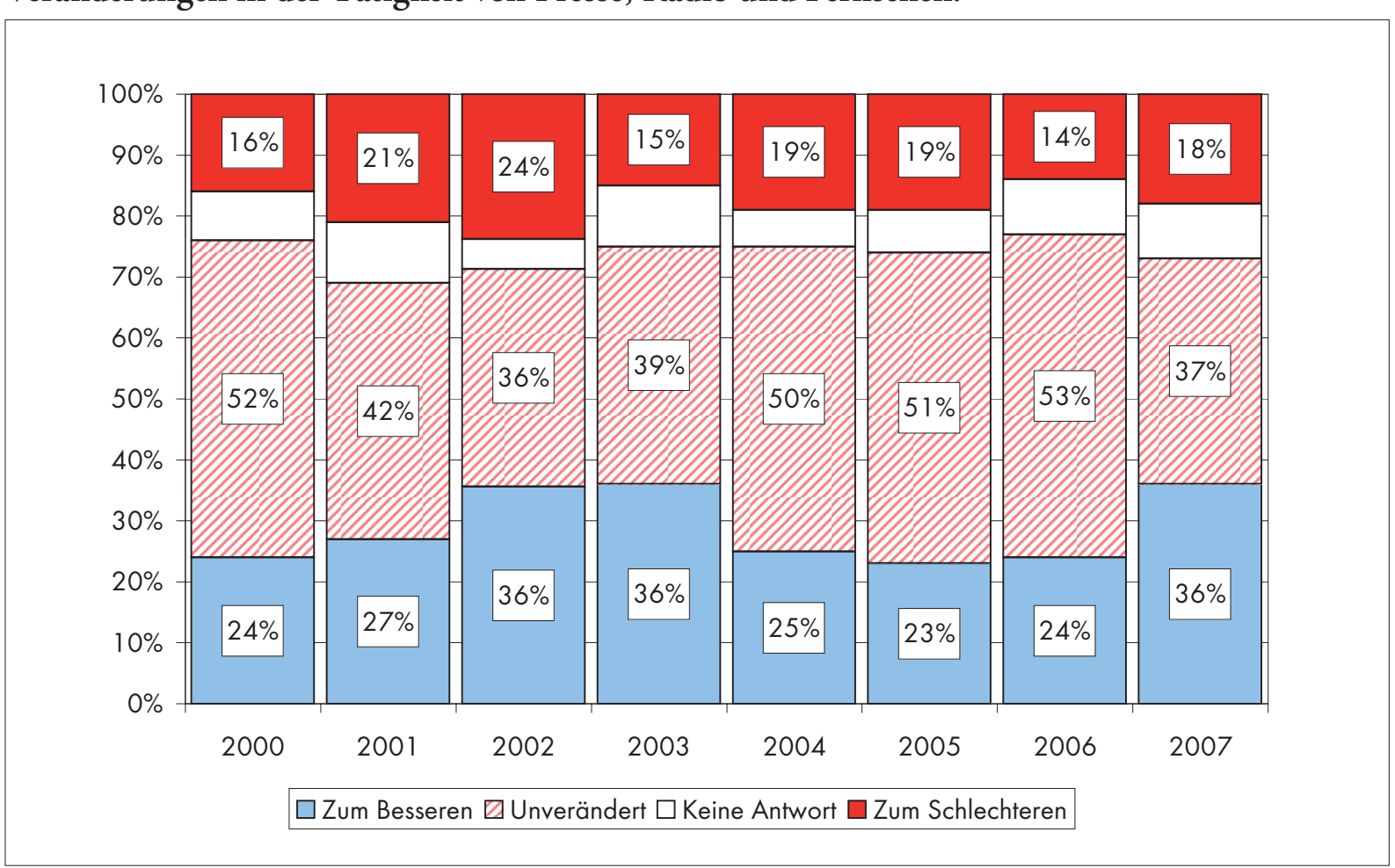

Quelle: Umfragen des Lewada-Zentrums, 20.-23. November 2007 http:// w w w.levada. ru./ press/200712 $0703 . \mathrm{html}$ 
Veränderungen in den Beziehungen zwischen Menschen verschiedener Nationalitäten?

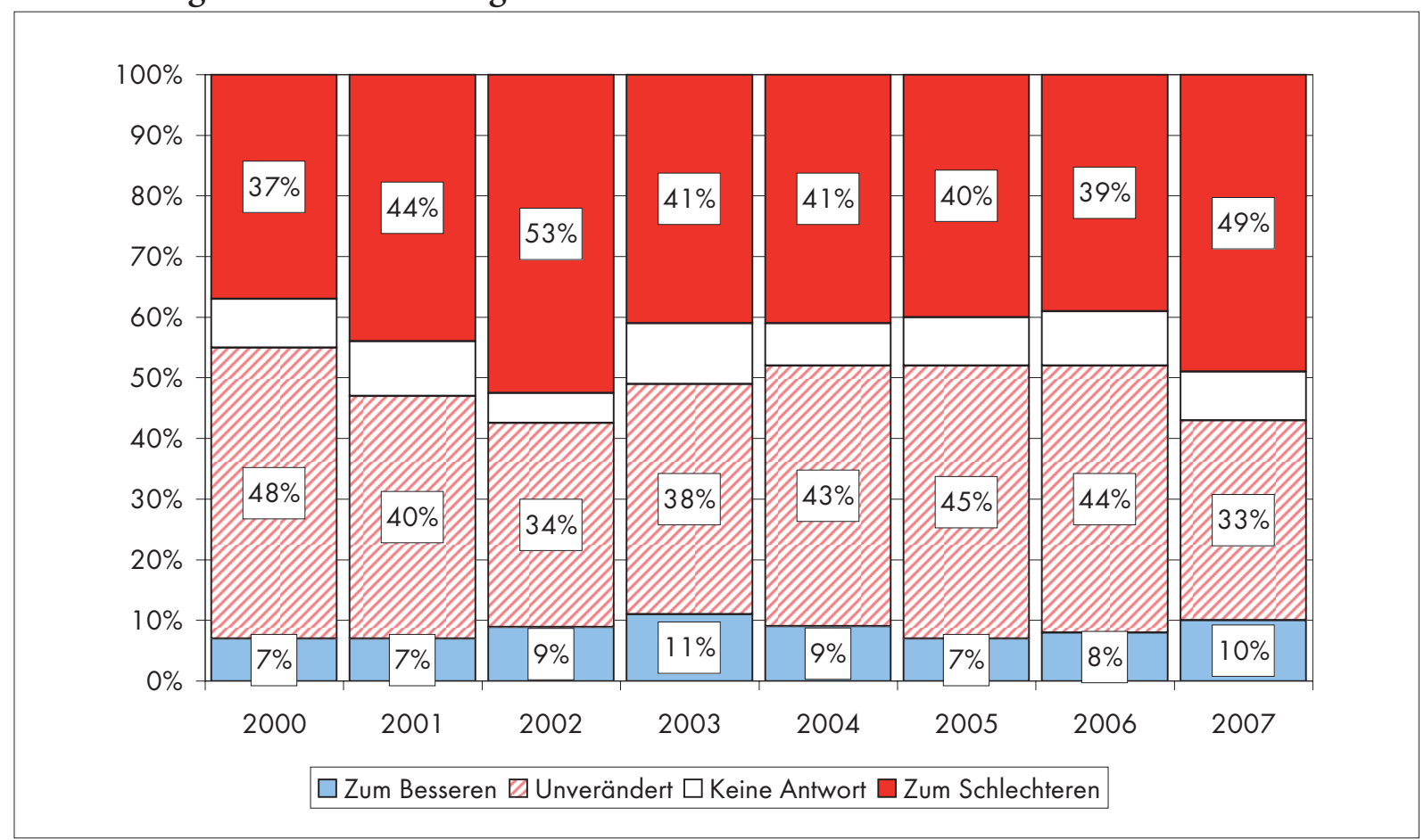

Veränderungen bei den Möglichkeit, gut zu verdienen?

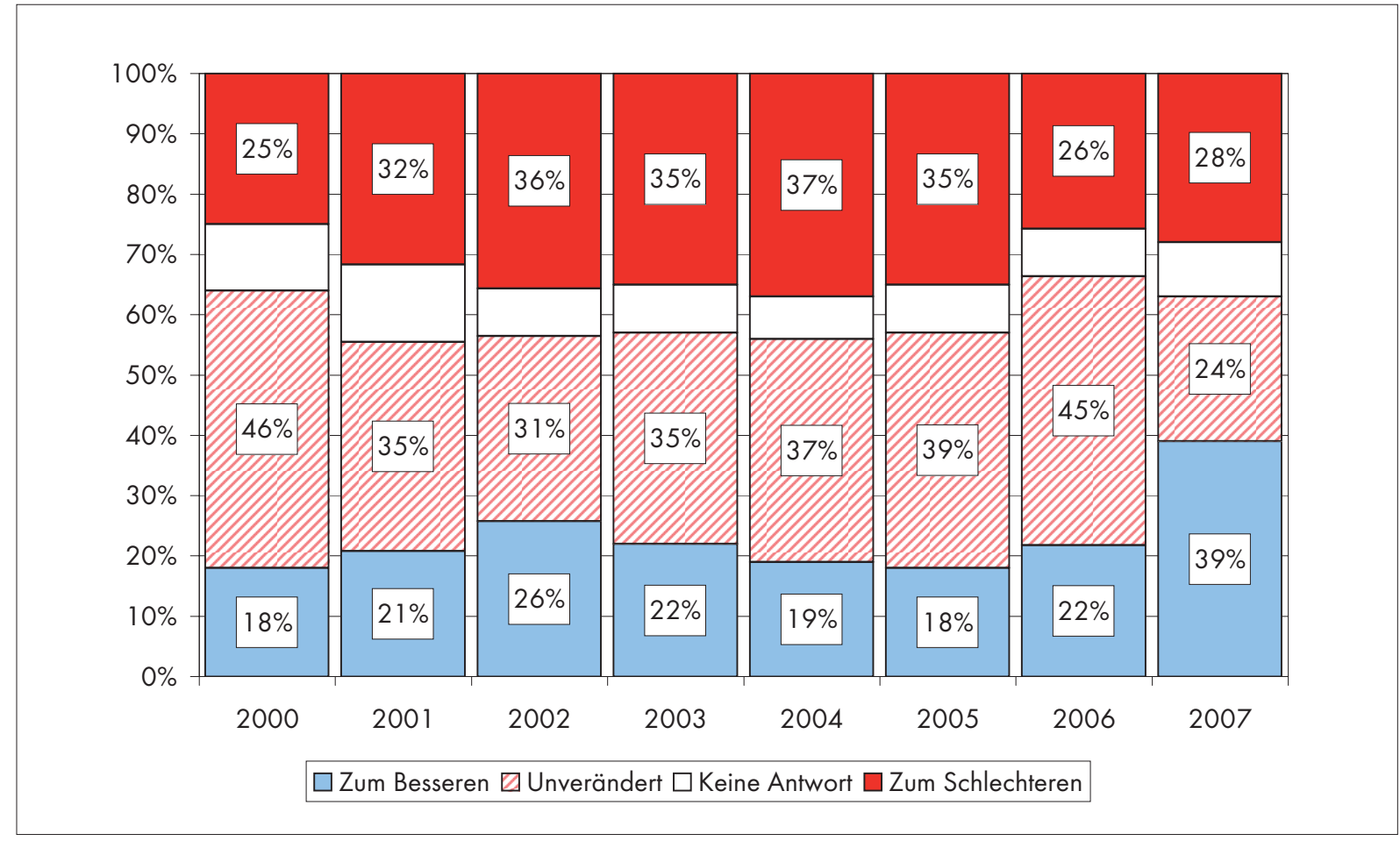


Veränderungen beim Einfluss einfacher Menschen auf staatliche Angelegenheiten?

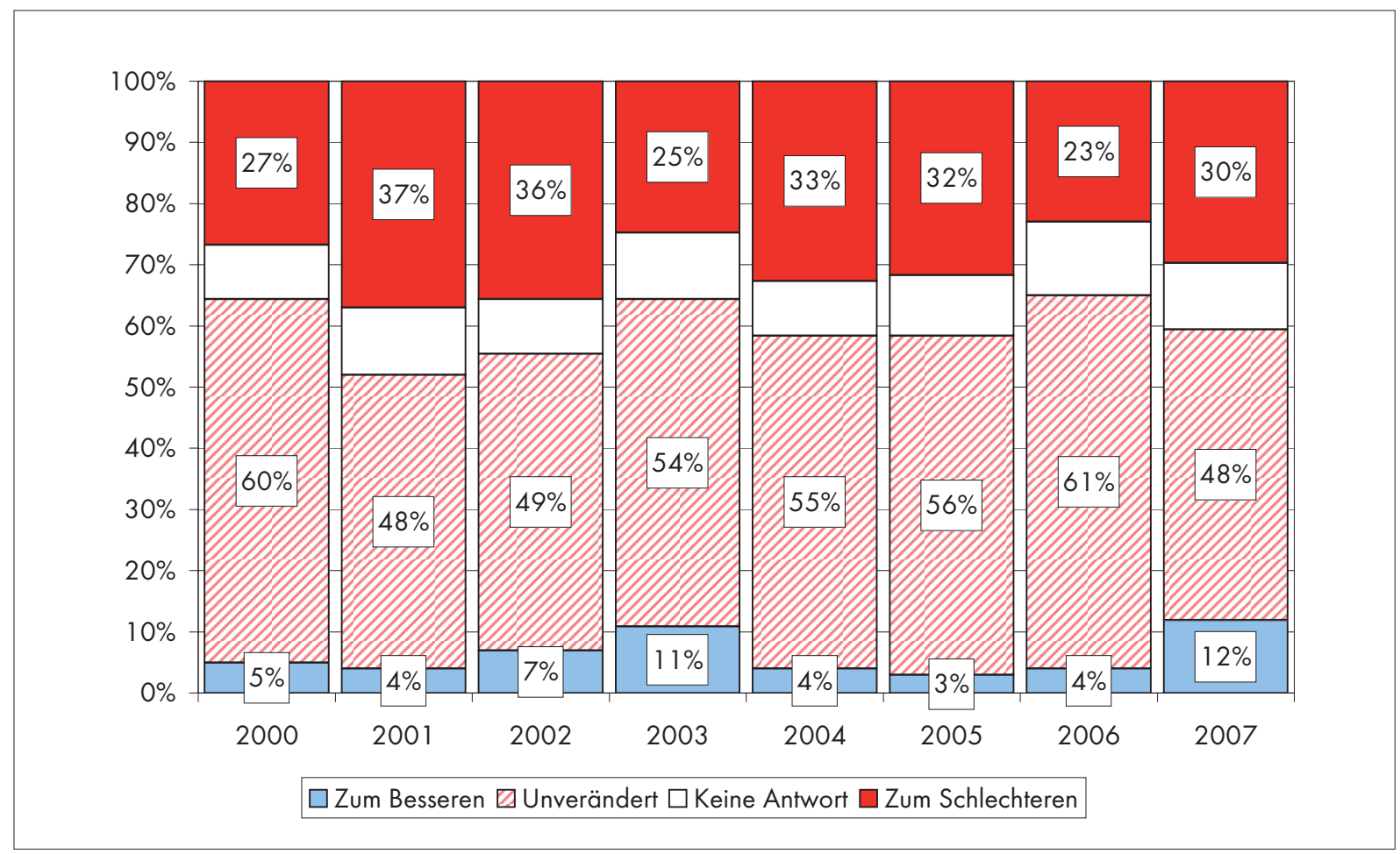

Veränderungen bei der Sicherheit für die Bürger?

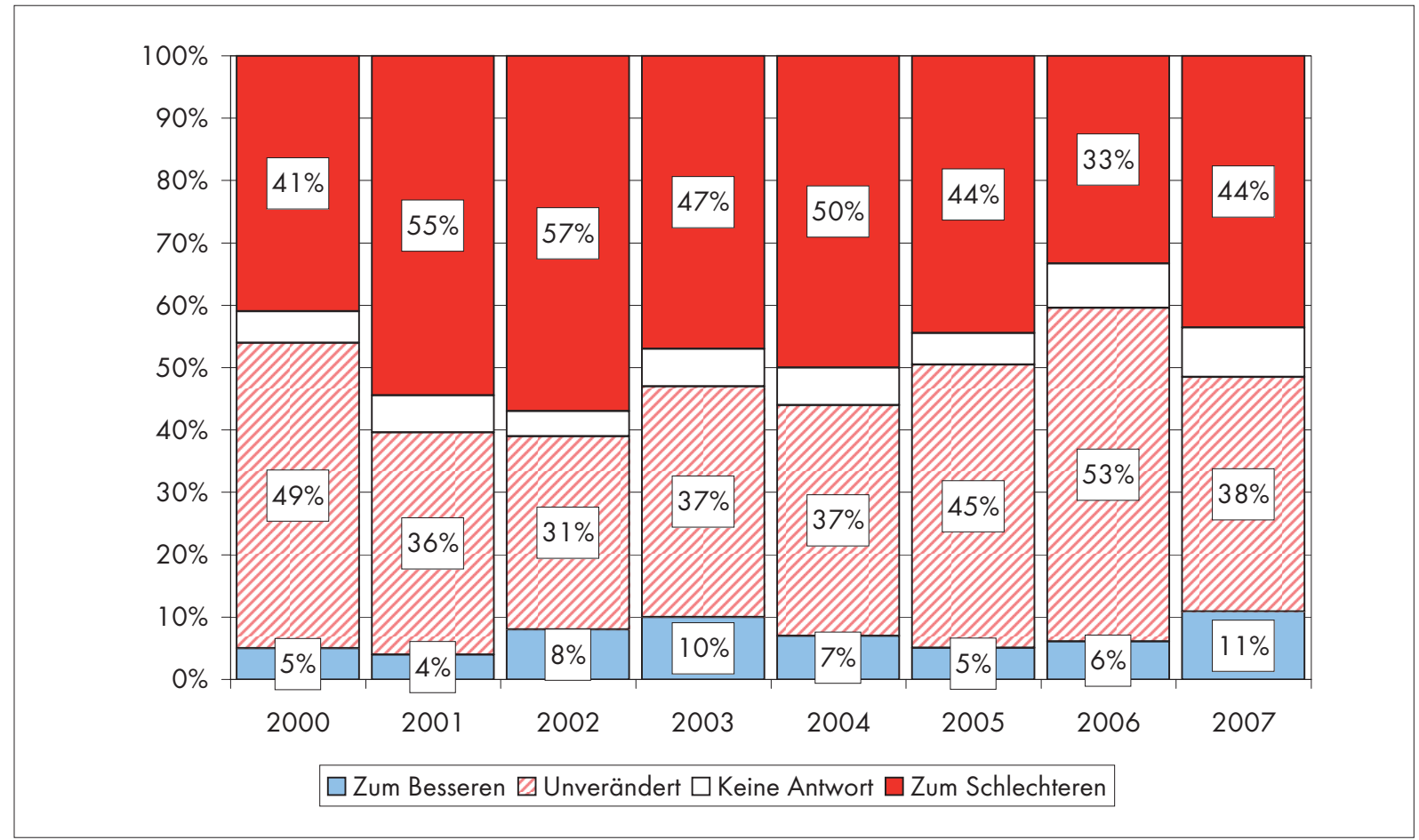

Quelle: Umfragen des Lewada-Zentrums, 20.-23. November 2007 http:// w w w.levada. ru./ press/200712 $0703 . \mathrm{html}$ 
Veränderungen bei der Möglichkeit, seine Meinung frei zu äußern?

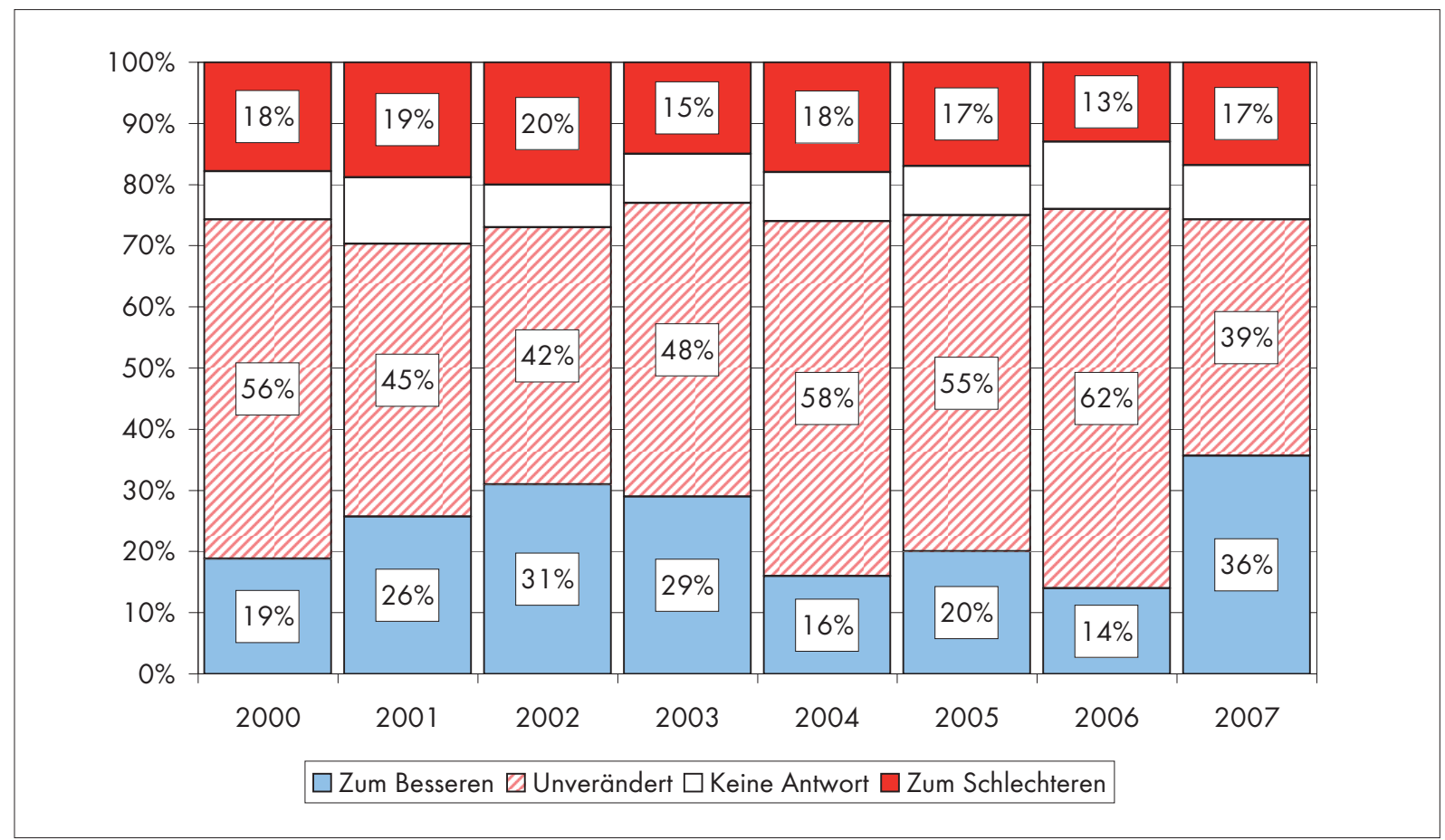

Veränderungen bei der gerechten Verteilung materieller Güter?

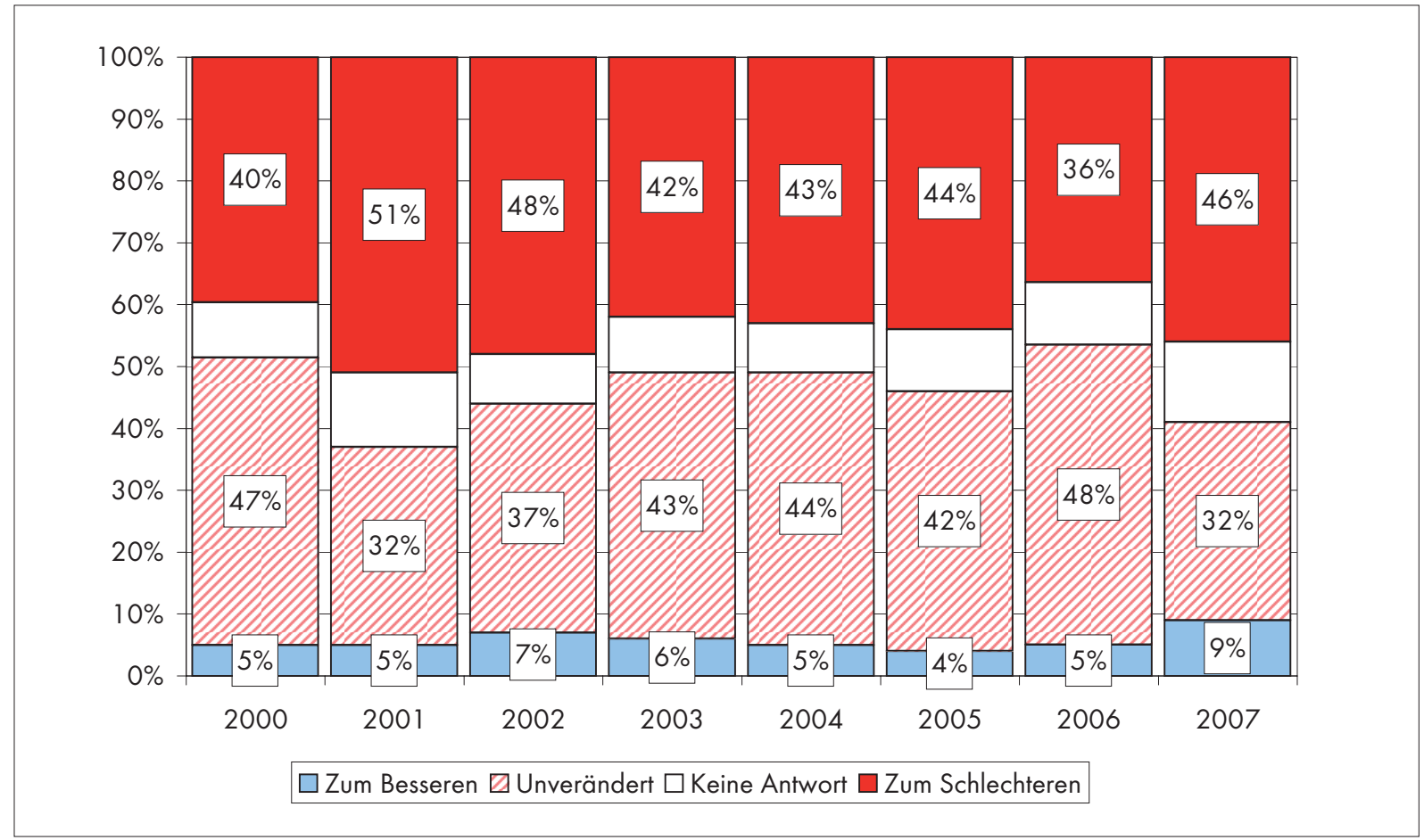

Quelle: Umfragen des Lewada-Zentrums, 20.-23. November 2007 http:// w w w.levada. ru./ press/200712 $0703 . \mathrm{html}$ 
Veränderungen in der Umweltsituation?

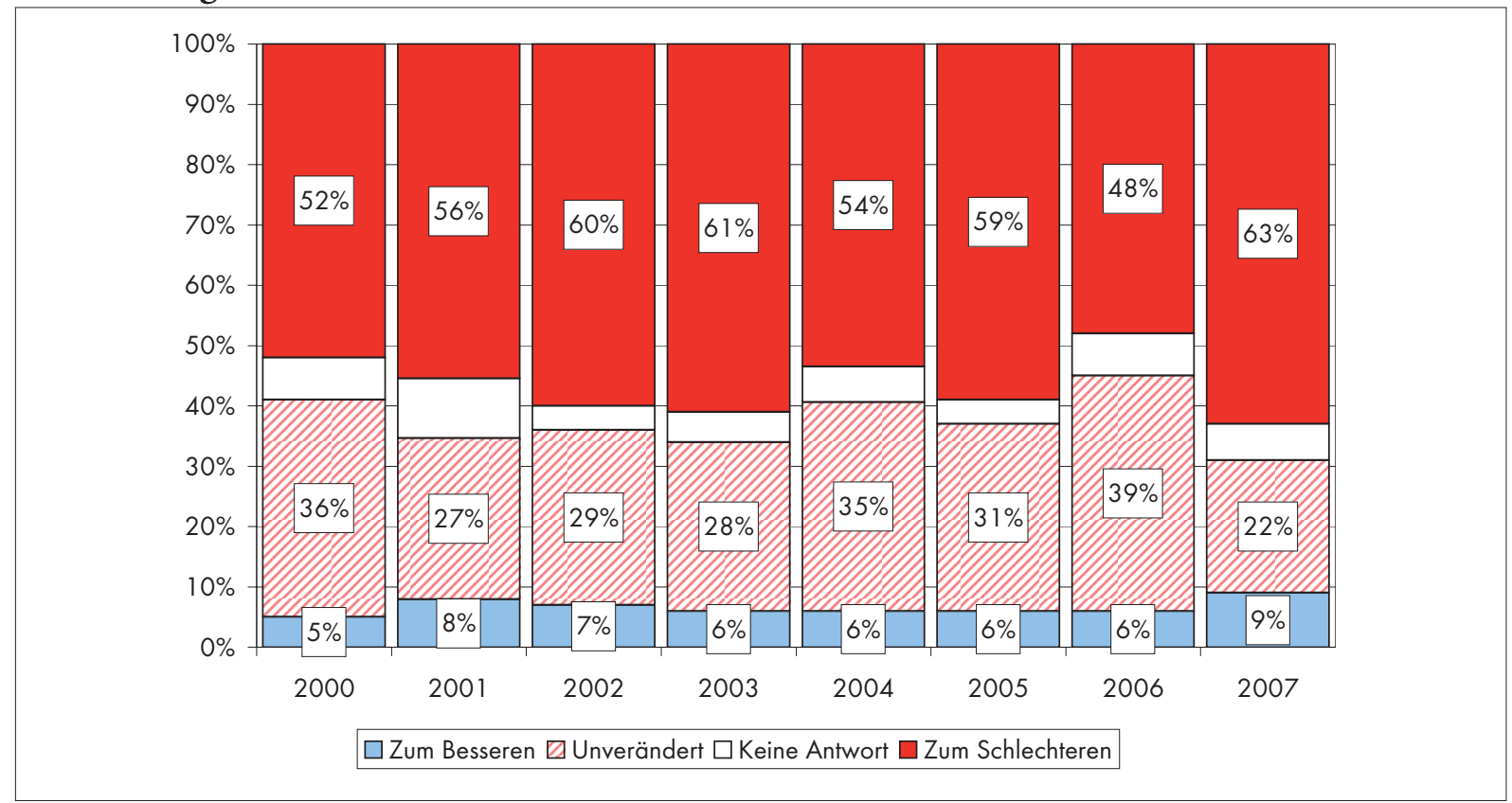

Quelle: Umfragen des Lewada-Zentrums, 20.-23. November 2007 http:// w w w.levada. ru./ p ress/200712 0703 .html

\section{Dokumentation}

\section{Die vorläufigen Ergebnisse der Präsidentenwahlen vom 2. März 2008}

Ungeachtet der Tatsache, dass der Wahlausgang lange vor dem 2. März feststand, sind die Wahlergebnisse von Interesse. Ziel des Wahlgangs war ja nicht die Auswahl eines Kandidaten im Rahmen eines politischen Wettbewerbs, sondern die Legitimierung des von den Eliten bestimmten Nachfolgers durch einen breiten, plebiszitären Prozess. In diesem Prozess können zugleich die regionalen Apparate ihre Loyalität demonstrieren und ihre Fähigkeit, die Bevölkerung zu mobilisieren. Insofern ist der Wahlgang auch eine Überprüfung der Funktionsfähigkeit der »Machtvertikale«. Die Ergebnisse zeigen erkennbare regionale Unterschiede.

Vorläufiges Ergebnis der russischen Präsidentenwahlen

(Stand: 5.3.2008, 11 Uhr, 85 Wahlkreise)

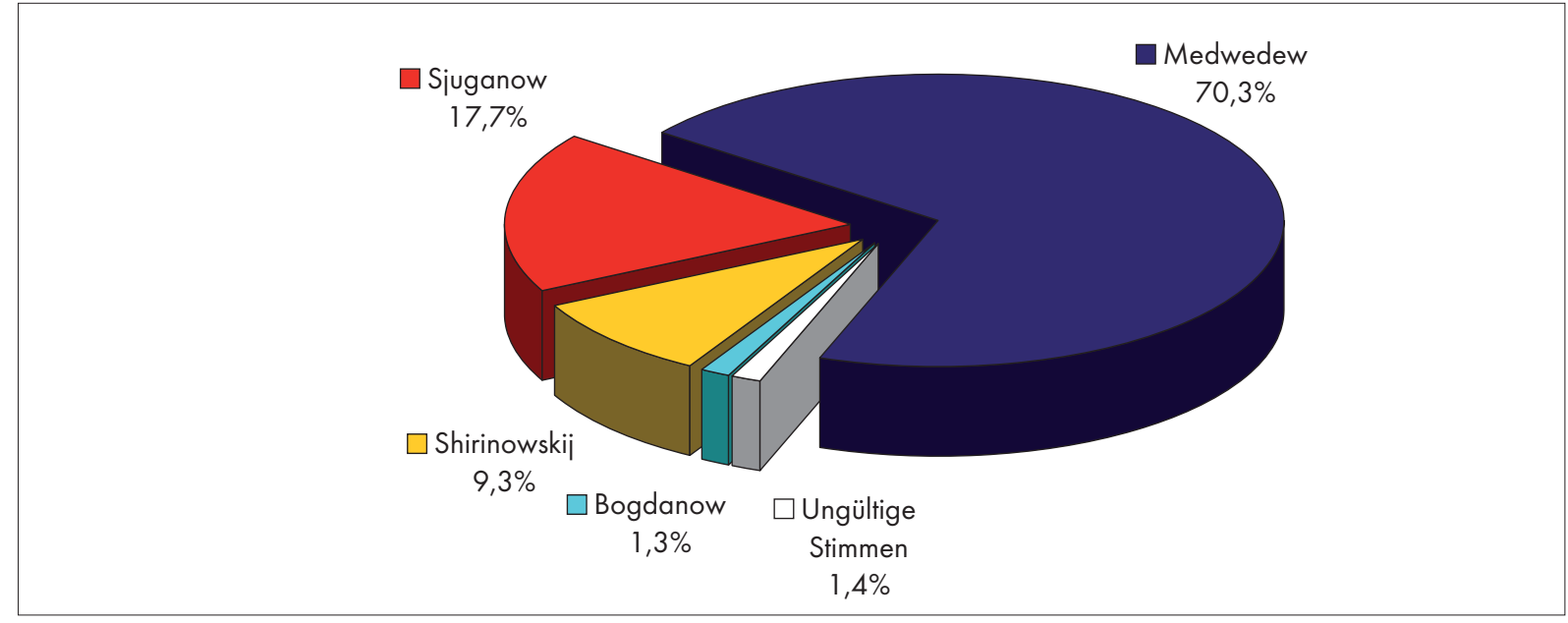

Quelle: http:// w w w.vybo ry.izbirko m.ru/ region/ regio n/ iz birko m? actio $n=$ sho $w \&$ ro ot=1\&tvd $=100100022249920 \&$ vrn $=100100022176412 \&$ reg io $n=0 \& \mathrm{~g}$ lo bal $=1 \&$ sub_regio $n=0 \&$ prver $=0 \&$ pronetvd $=$ null $\&$ vibid $=100100022249920 \&$ ty pe $=226,5$. März 2008 
Wahlprotokoll. Vorläufiges Ergebnis der Präsidentenwahlen vom 2.3.2008

Stand: 5.3.2008, 11 Uhr, 85 Wahlbezirke

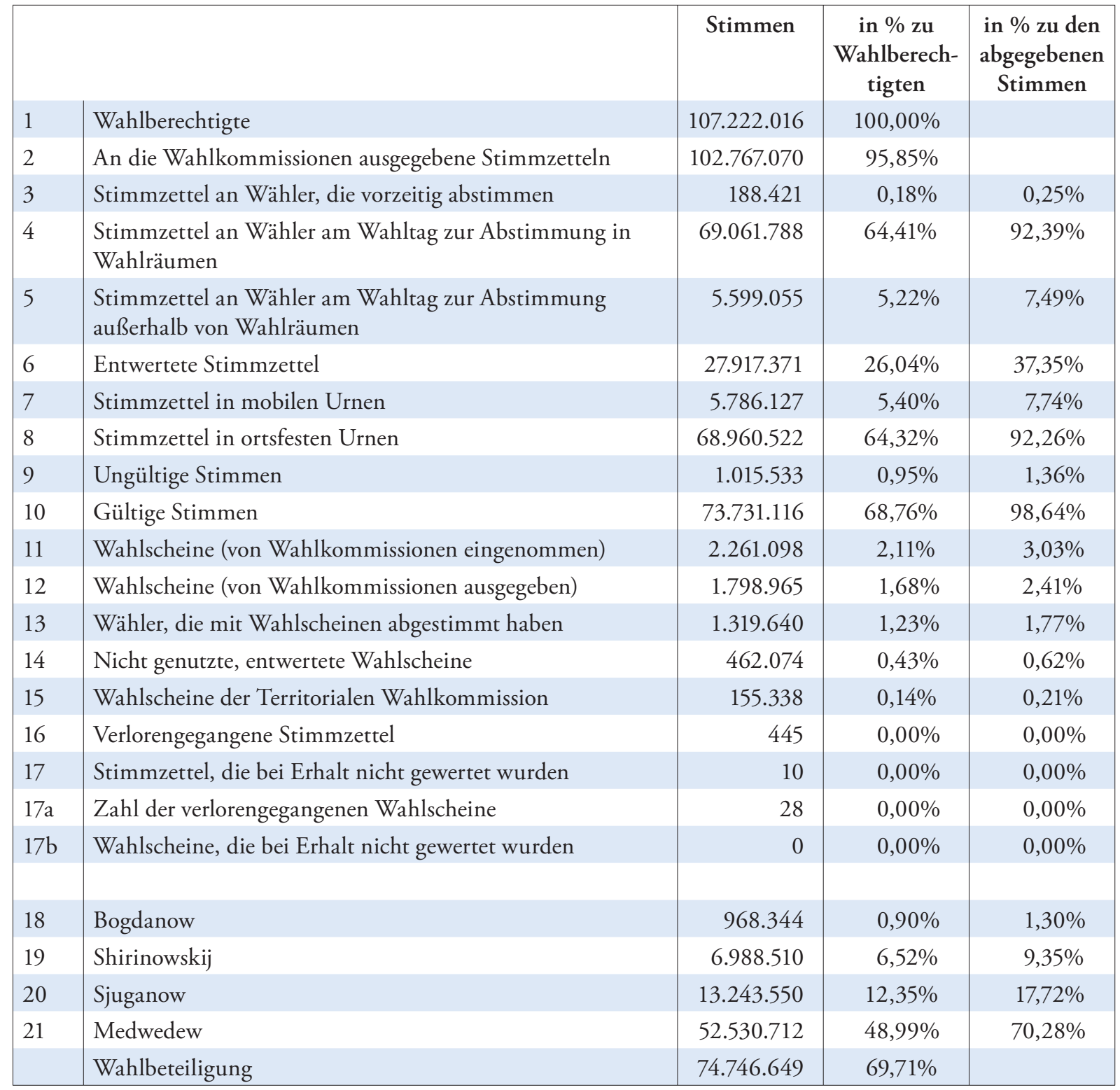

Quelle: http:// w w w.vybory.izbirkom.ru/ region/ region/

izb irko $m$ ? a ctio $n=s h o w \&$ ro ot $=1 \&$ tvd $=100100022249920 \&$ vrn $=100100022176412 \&$ reg ion $=0 \& \mathrm{~g}$ lo bal $=1 \&$ sub_ reg ion $=0$ \& prver $=0 \& p$ ronetvd $=$ null $\&$ vibid $=100100022249920 \&$ type $=226,5$. März 2008 




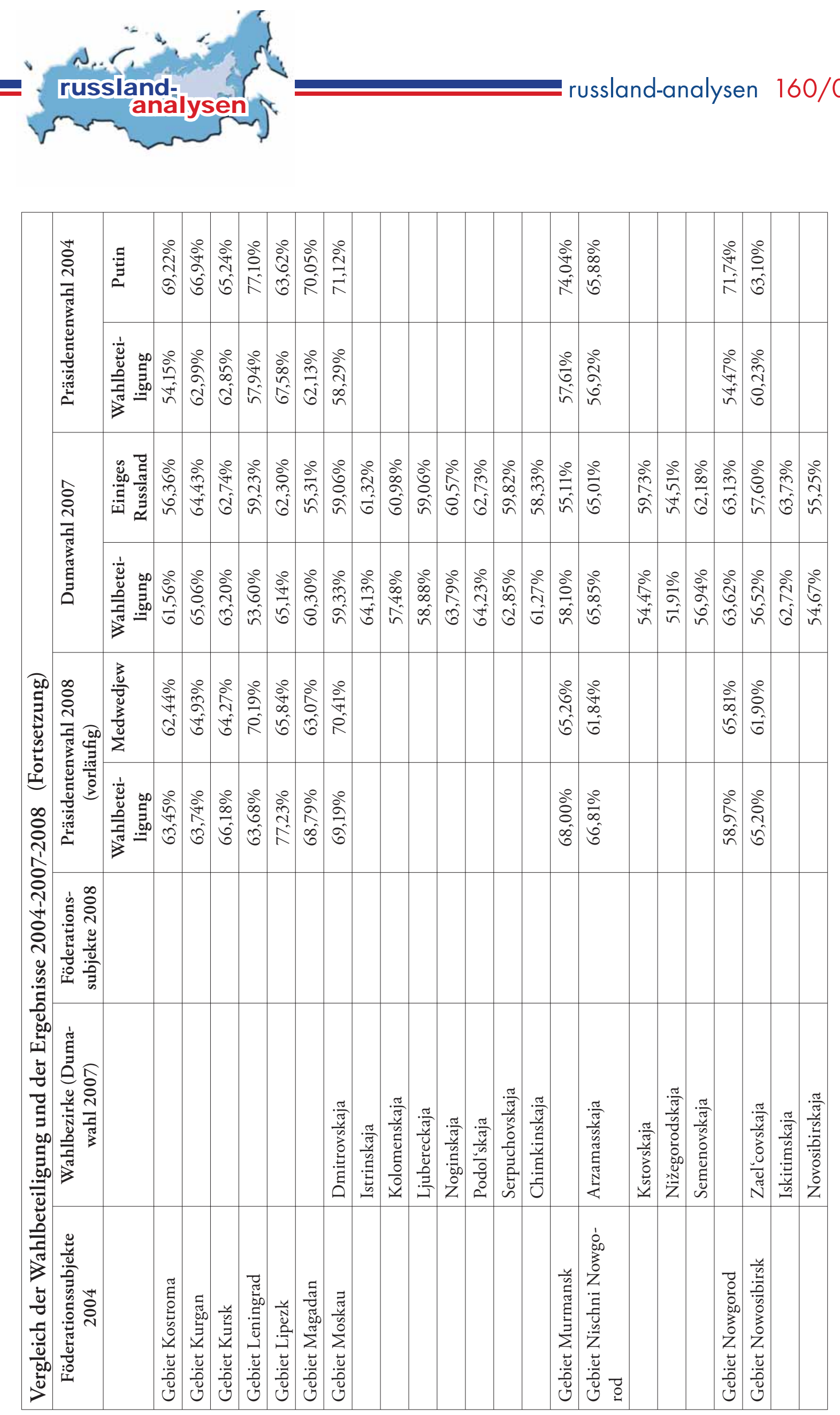






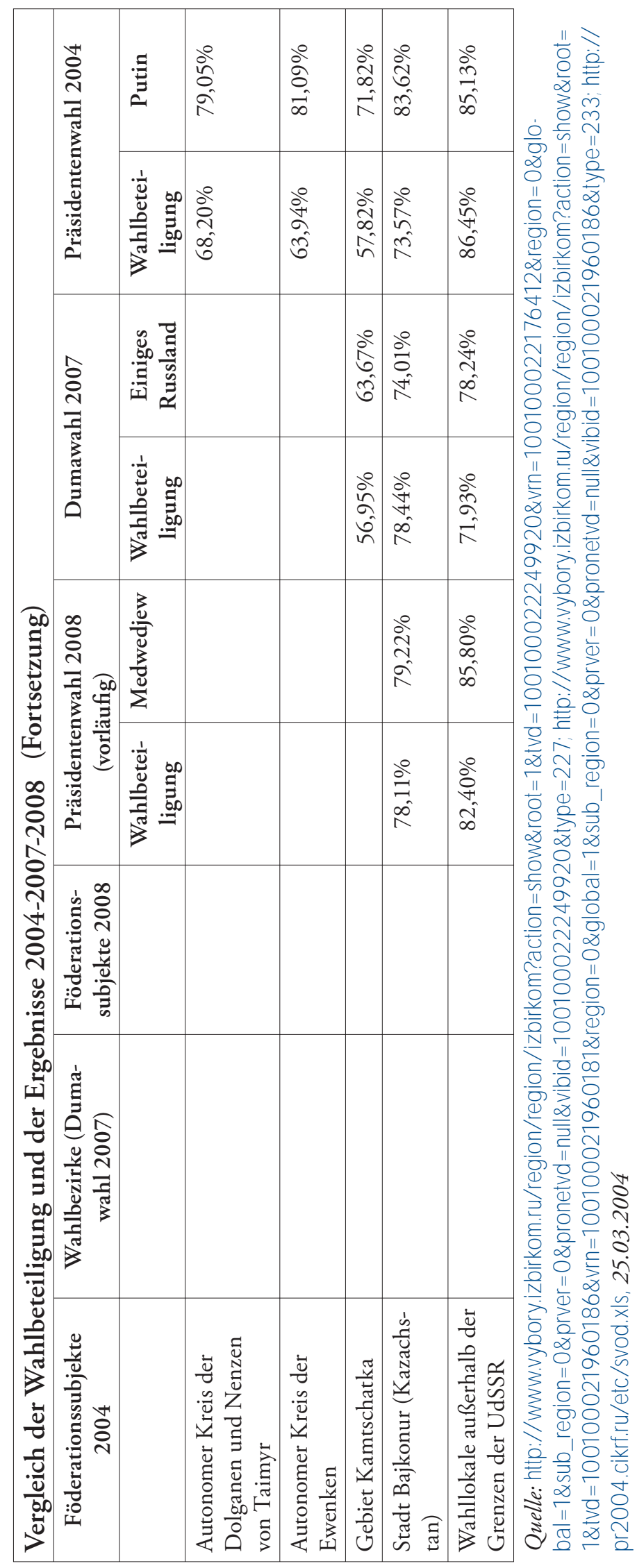




\section{Umfrage}

\section{Braucht Russland internationale Wahlbeobachter?}

Die russischen Wahlbehörden haben auch für die Präsidentenwahlen am 2. März 2008 ausländische Wahlbeobachter eingeladen, haben ihre Zahl aber auf 400 begrenzt. Das Kontingent der OSZE wurde auf 70 begrenzt. Das ODIHR (Büro für demokratische Institutionen und Menschenrechte), die OSZE-Behörde, die Wahlbeobachtungen organisiert, entschied sich, unter den gegebenen Bedingungen keine Beobachtermission zu entsenden - eine Entscheidung, die von der russischen Seite kritisiert wurde.

\section{Braucht man bei Wahlen in Russland internationale Wahlbeobachter?}

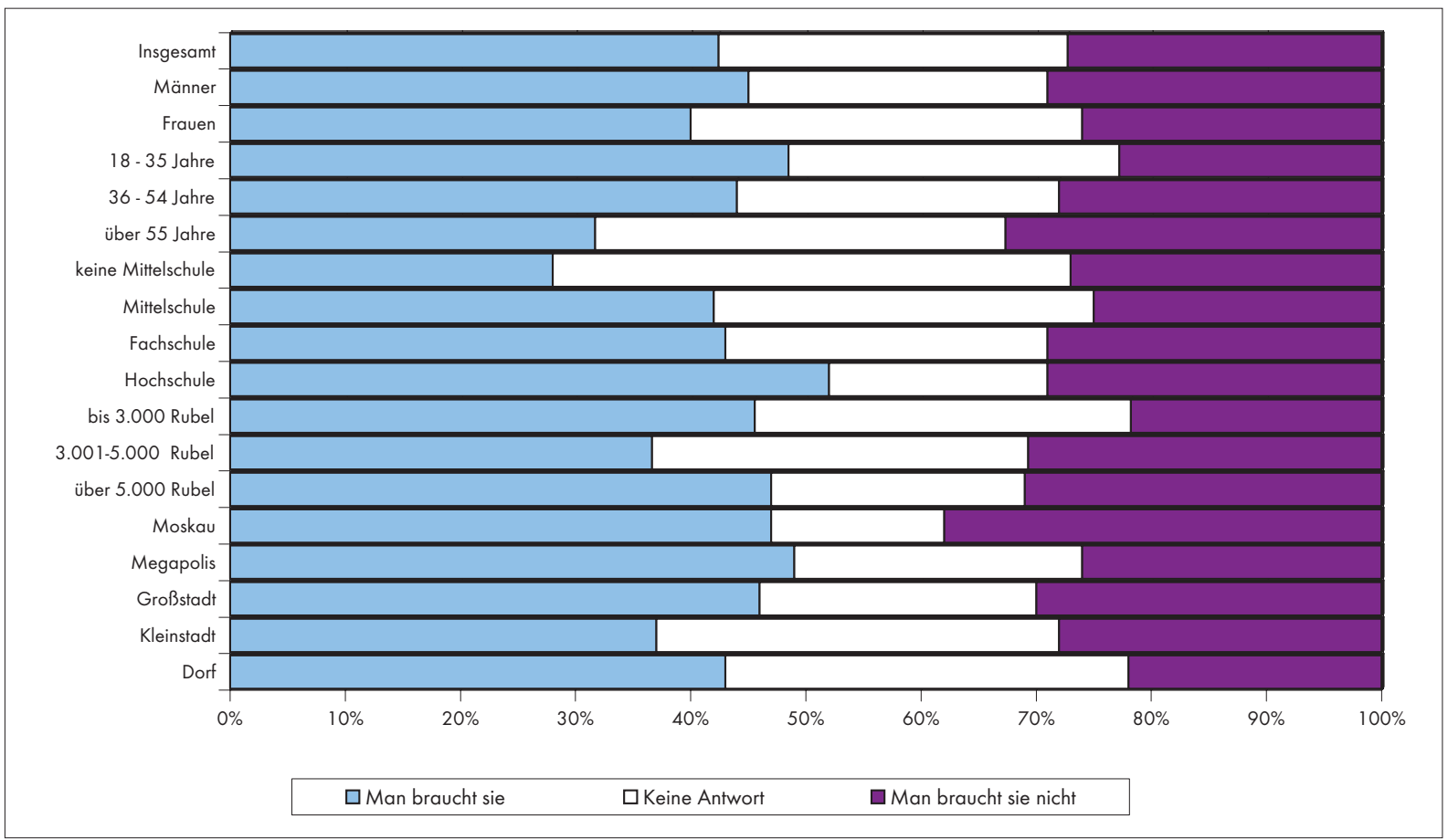

Quelle: Umfrage der "Stiftung Öffentliche Meinung"(FOM) vom 16.-17.2.2008

http:// bd fo m.ru/ report/ map/dominant/ dom0808/d080822 
Wissen Sie, dass die OSZE es abgelehnt hat, Vertreter zur Beobachtung der bevorstehenden Wahlen in Russland zu entsenden?

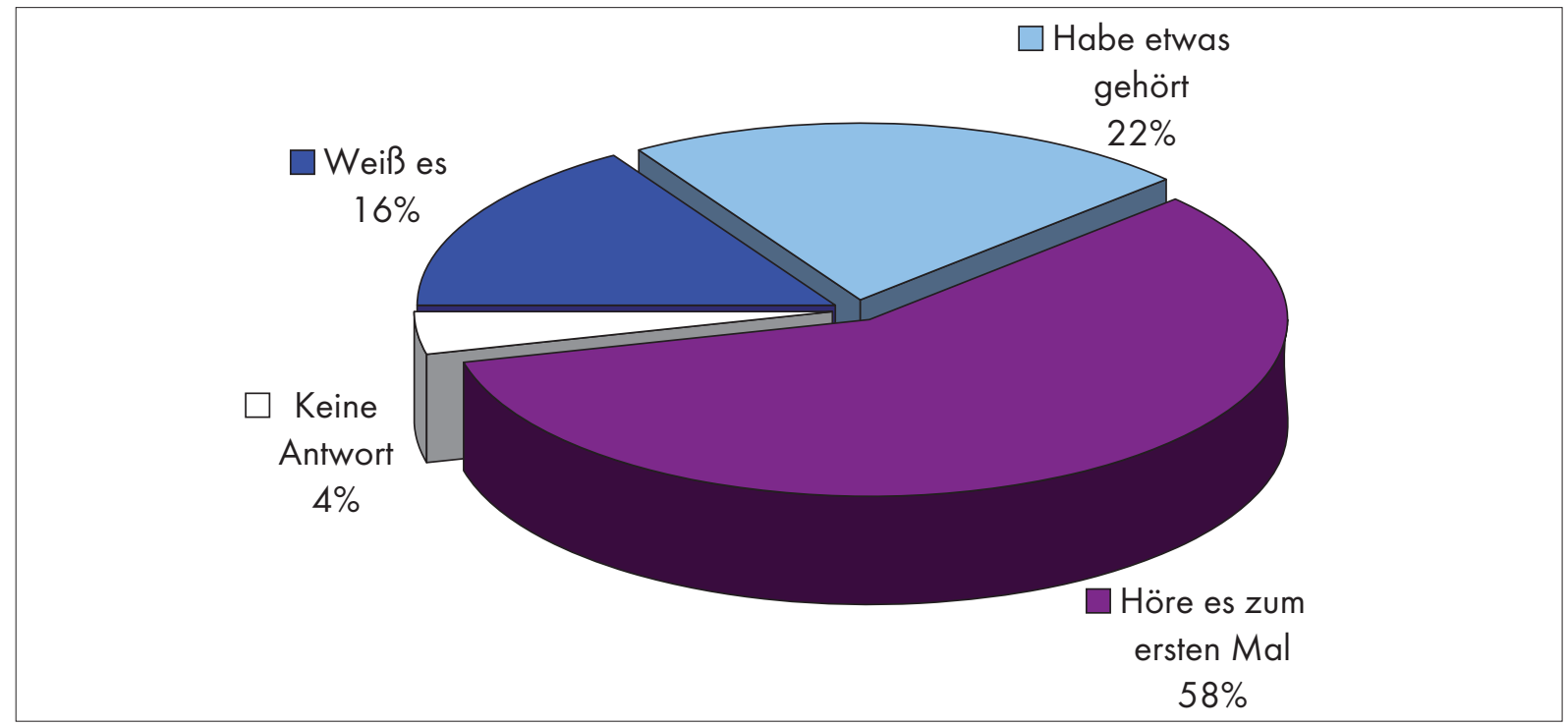

Wäre es besser oder schlechter, wenn OSZE-Beobachter bei den Präsidentschaftswahlen anwesend wären?

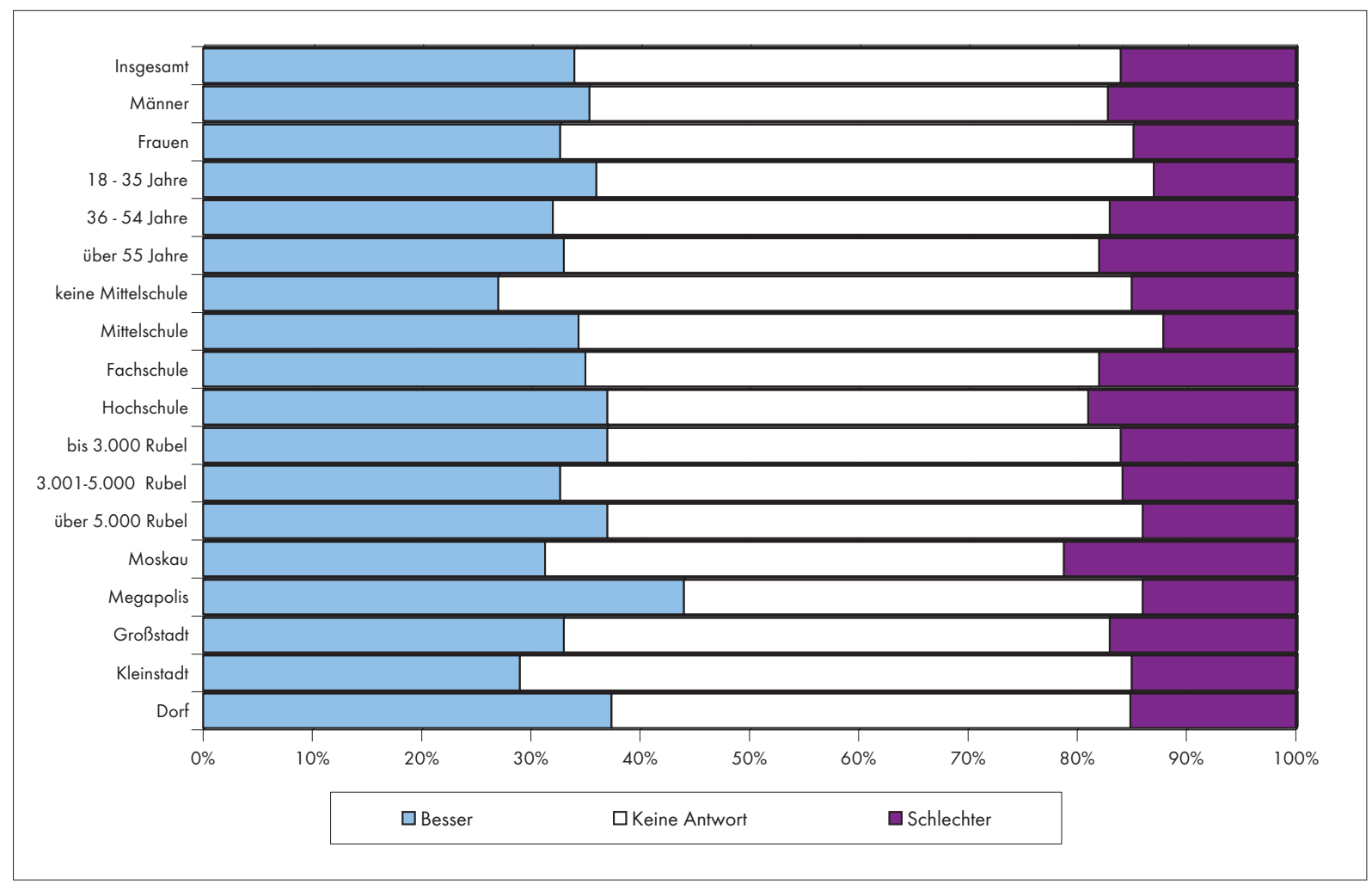

Quelle: Umfrage der "Stiftung Öffentliche Meinung" (FOM) vom 16.-17.2.2008

http:// bd.fom.ru/ report/ map/ do minant/ dom0808/ d080822 


\section{Umfrage}

\section{Die öffentliche Meinung zu Frauenrechten im internationalen Vergleich}

Wie wichtig ist es Ihrer Meinung nach für Frauen, gegenüber Männern vollständig gleichberechtigt zu sein? (sehr wichtig, etwas wichtig, nicht sehr wichtig, unwichtig)

Anteil von "sehr wichtig"

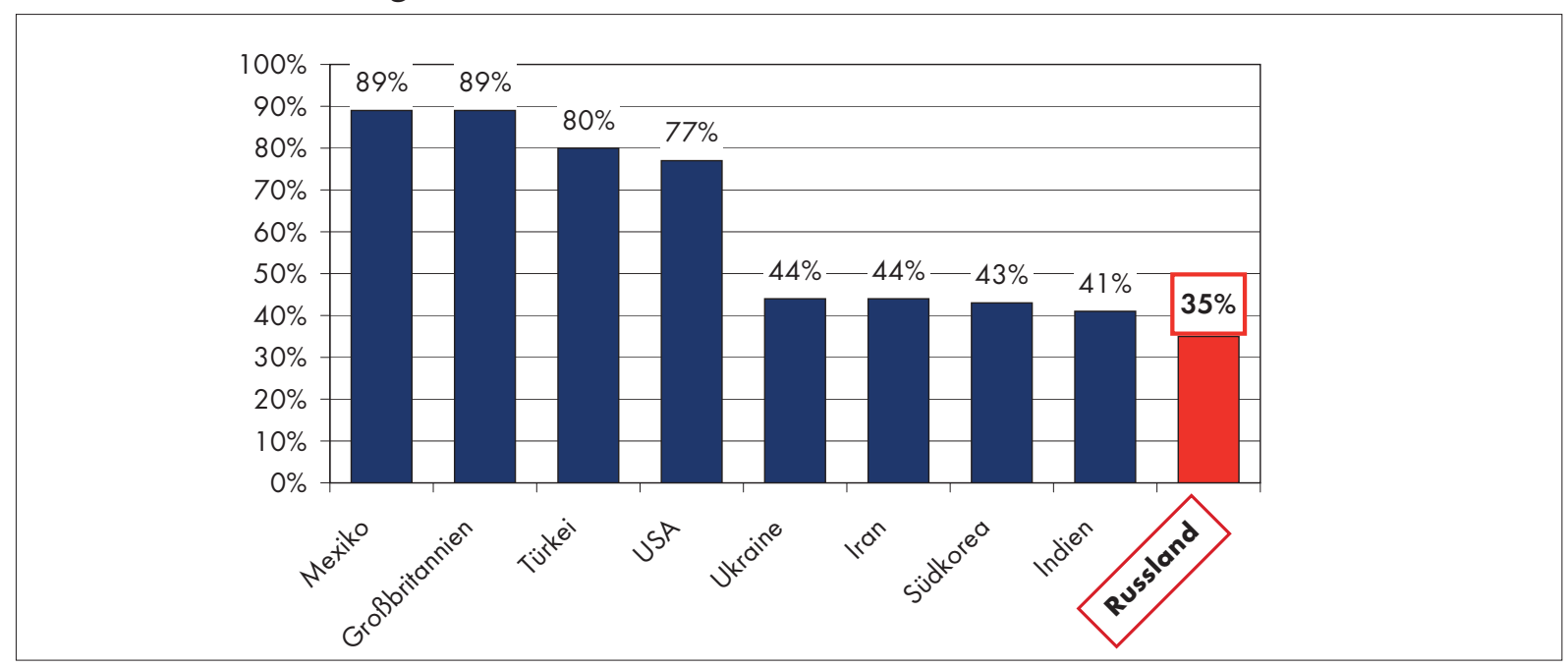

Wenn Sie in Ihrem Leben zurückblicken, würden Sie sagen, dass Frauen im Vergleich zu Männern heutzutage in der Gesellschaft deutlich stärker gleichberechtigt sind, etwas mehr gleichberechtigt, etwas weniger gleichberechtigt oder deutlich weniger gleichberechtigt sind? Anteil von »deutlich stärker gleichberechtigt« sowie von keine Veränderung oder weniger Gleichberechtigung

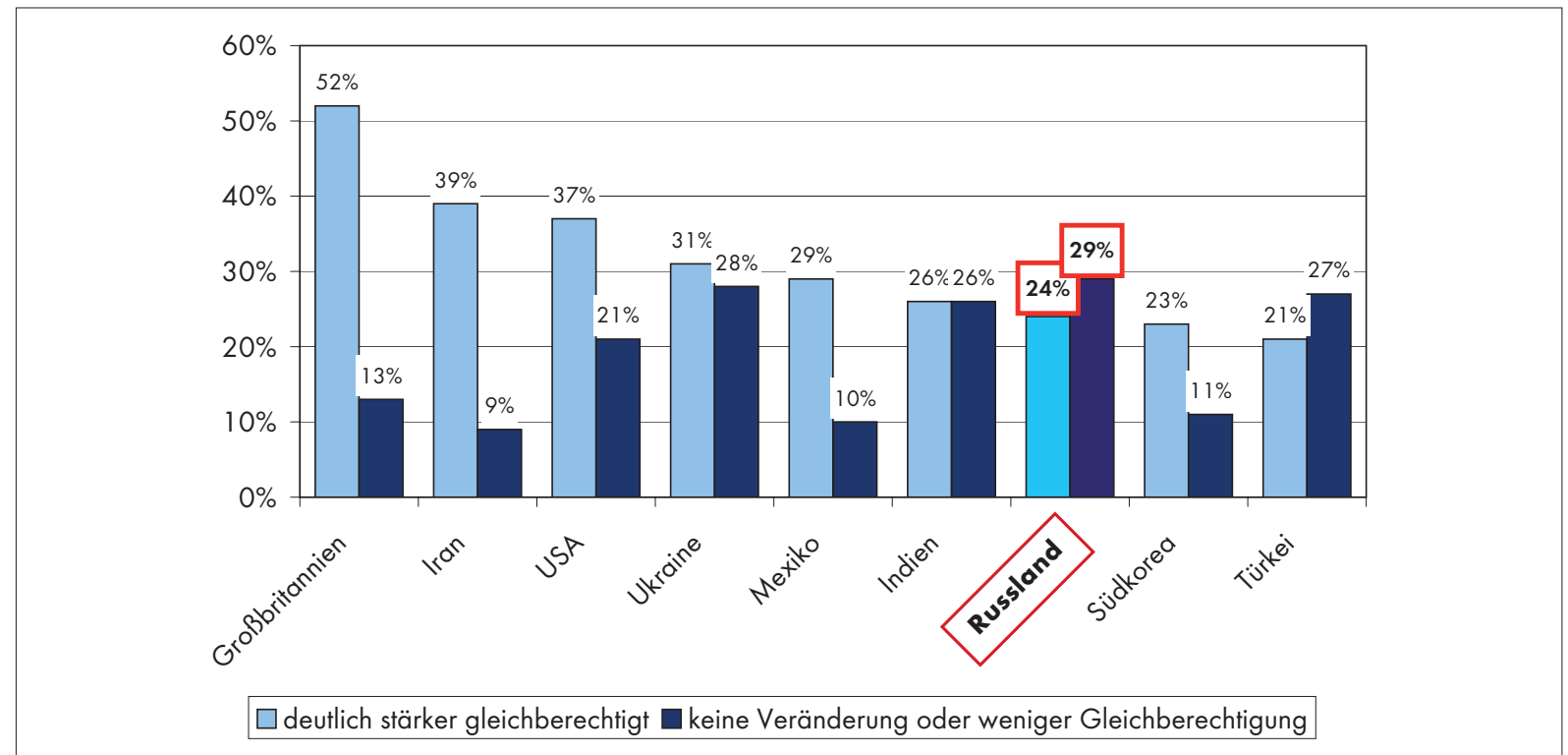

Quelle: Repräsentative Umfrage unter der Bevölkerung von 16 Ländern im Januar und Februar 2008 durch WorldPublicOpinion.org unter Leitung des Program on International Policy Attitudes (PIPA) der University of Maryland (USA). Die Umfrage in Russland wurde vom Levada Zentrum durchgeführt.

http:// w w w.worldpublicopinion.org/pipa/pdf/ mar08/ W P0_Women_M ar08_quaire_emb.pdf 
Finden Sie, dass die Regierung Maßnahmen gegen die Diskriminierung von Frauen ergreifen sollte oder dass dies nicht Aufgabe der Regierung ist?

Zustimmung zu Regierungsverantwortung

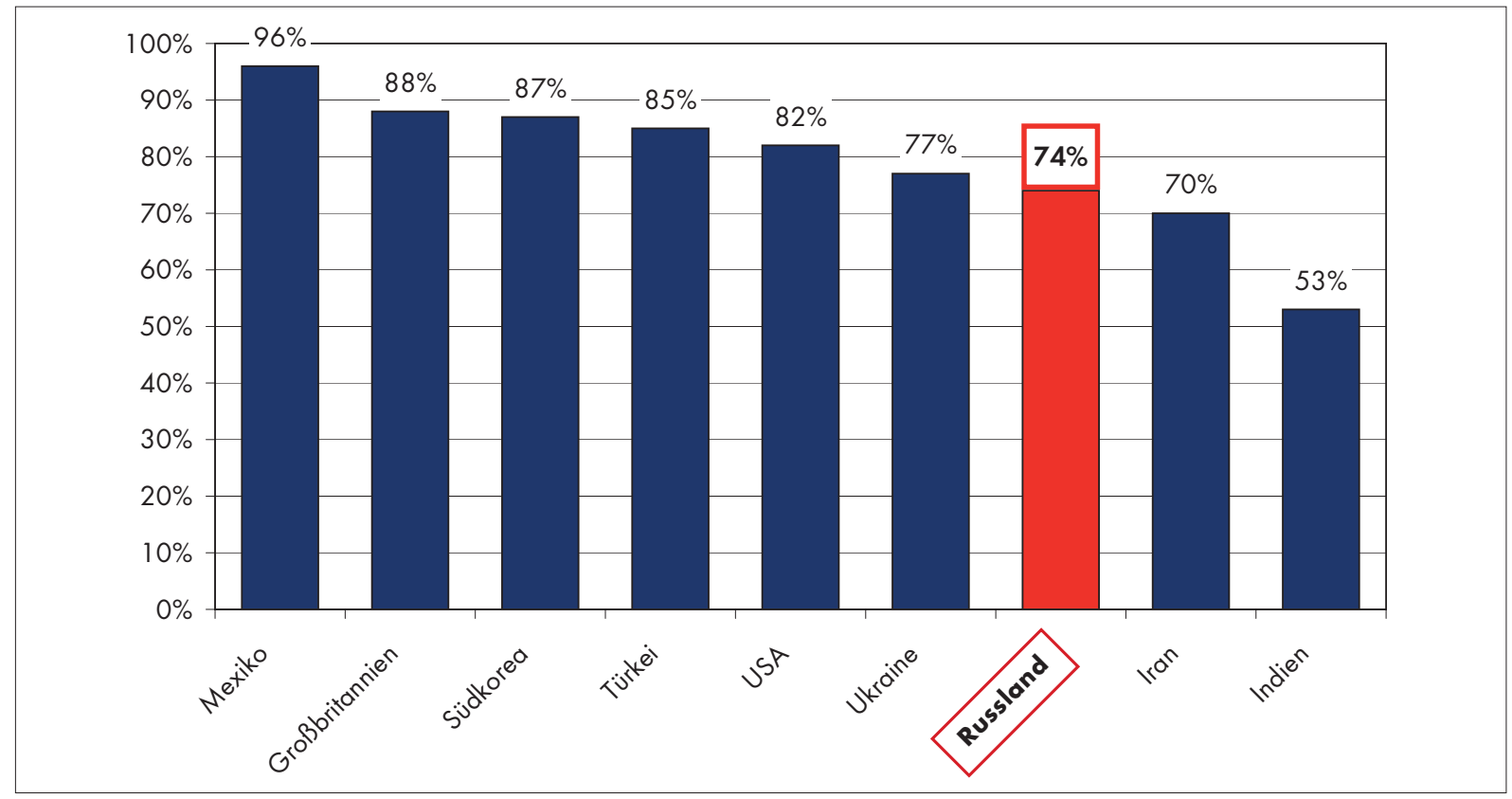

Finden Sie, dass die Regierung genug gegen die Diskriminierung von Frauen tut, oder meinen Sie, dass sie mehr tun sollte?

Anteil von "Sie sollte mehr tun«

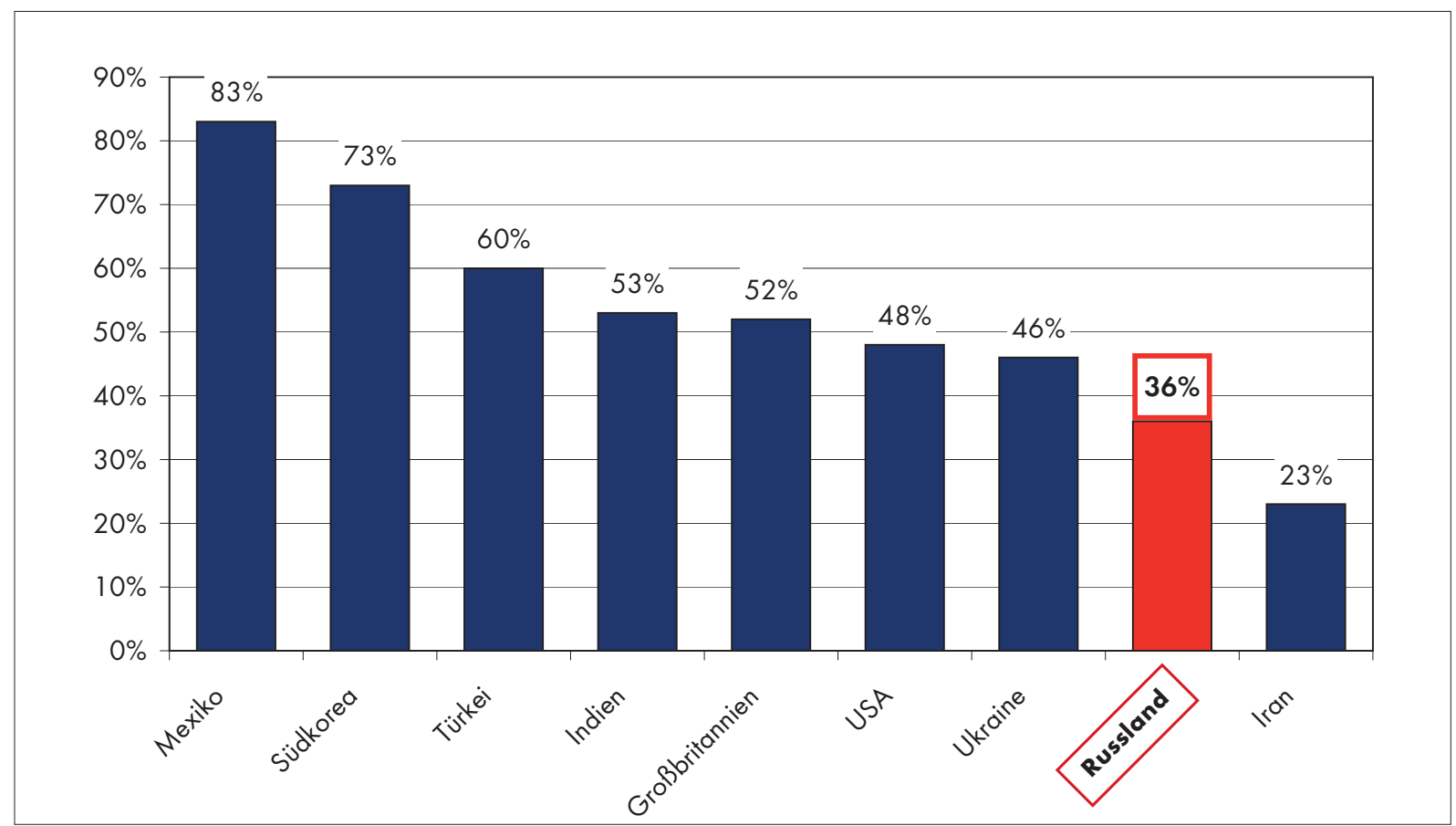

Quelle: Repräsentative Umfrage unter der Bevölkerung von 16 Ländern im Januar und Februar 2008 durch WorldPublicOpinion.org unter Leitung des Program on International Policy Attitudes (PIPA) der University of Maryland (USA). Die Umfrage in Russland wurde vom Levada Zentrum durchgeführt.

http:// w w w.worldpublicopinion.org/pipa/pdf/ mar08/ W PO_W omen_M ar08_quaire_emb.pdf 


\section{Chronik}

\section{Vom 29. Februar bis zum 6. März 2008}

\begin{tabular}{|c|c|}
\hline 29.2 .2008 & en. \\
\hline 2.3 .2008 & $\begin{array}{l}\text { Bei den Präsidentenwahlen erhält Dmitrij Medwedjew 70,2 \% der abgegebenen Stimmen. Der kommunistische Kan- } \\
\text { didat Gennadij Sjuganow folgt mit } 17,7 \% \text { und Wladimir Shirinowskij mit 9,4 \%. Der >liberale Kandidat Andrej } \\
\text { Bogdanow erhält 1,3\%. Die Wahlbeteiligung liegt bei } 67 \% \text {. } \\
\text { Die Wahlbeobachter der GUS und der Shanghai Organisation bewerten den Urnengang positiv. Vertreter des Euro- } \\
\text { parats und der Parlamentarischen Versammlung der OSZE äußern sich skeptischer }\end{array}$ \\
\hline 2.3 .2008 & $\begin{array}{l}\text { In } 11 \text { Föderationssubjekten finden Wahlen für die regionalen Parlamente statt: Gebiet Rostow, Gebiet Uljanowsk, } \\
\text { Gebiet Amur, Gebiet Jaroslawl, Gebiet Iwanowo, Gebiet Swerdlowsk, Altaj-Region, Republik Sacha (Jakutien), } \\
\text { Republik Baschkortostan, Republik Inguschetien, Republik Kalmykien. }\end{array}$ \\
\hline 3.3 .2008 & $\begin{array}{l}\text { In Petersburg verhaften Sicherheitskräfte Maksim Resnik, einen prominenten Jabloko-Politiker, der zu den Orga- } \\
\text { nisatoren der Märsche der Nichteinverstandenen gehört, unter der Beschuldigung, er habe einen Polizeibeamten } \\
\text { angegriffen. Dies wird von Zeugen bestritten. Am 4.März verurteilt ihn ein Petersburger Gericht zu zwei Mona- } \\
\text { ten Haft. }\end{array}$ \\
\hline 3.3 .2008 & $\begin{array}{l}\text { i einem Treffen mit Dmitrij Medwedjew bestätigt Putin seine Absicht, unter dem Präsidenten Medwedjew Minis- } \\
\text { rpräsident zu werden. }\end{array}$ \\
\hline 3.3 .2008 & $\begin{array}{l}\text { In Moskau unterbinden Polizeikräfte einen »Marsch der Nichteinverstandenen«, mit dem Oppositionelle gegen die } \\
\text { Wahlen protestieren wollen. In St. Petersburg nehmen ca. } 1.000 \text { Personen an einem ähnlichen Protestmarsch teil, } \\
\text { der von den Behörden genehmigt worden war. }\end{array}$ \\
\hline 3.3 .2008 & $\begin{array}{l}\text { Der Erdgaskonzern Gazprom drosselt die Erdgaslieferungen in die Ukraine, nachdem die Abmachungen zwischen } \\
\text { Putin und Juschtschenko vom 12./13.2. bisher nicht in die Tat umgesetzt und die ausstehenden Zahlungen nicht } \\
\text { geleistet wurden. Ein Gazpromsprecher erklärt, dass etwa um } 25 \% \text { weniger Erdgas in die Ukraine fließen werden. } \\
\text { Der staatliche ukrainische Energiekonzern Naftogaz Ukrainy teilte später mit, dass Gazprom seine Lieferungen um } \\
35 \% \text { reduziert habe, was einem geringeren Volumen von } 46 \text { Mio. } \mathrm{m}^{3} \text { täglich entspricht. Nach Europa fließen täg- } \\
\text { lich rund } 410 \mathrm{Mio} \mathrm{m}^{3} \text { russisches Erdgas. }\end{array}$ \\
\hline 4.3 .2007 & $\begin{array}{l}\text {, dass das Ministerium für Information und Technologie alle Mobilfunk- } \\
\text { angewiesen hat, dem Inlandsgeheimdienst FSB ungehinderten Zugang für } \\
\text { etkommunikation zu gewähren. }\end{array}$ \\
\hline 4.3 .2007 & $\begin{array}{l}\text { nen in einem Protestzug zur } \\
\text { n. }\end{array}$ \\
\hline 4.3 .2007 & rlichen Pressekonferenz die \\
\hline 5.3.2007 & $\begin{array}{l}\text { Die Tageszeitung "Kommersant« meldet, dass die Generalstaatsanwaltsc } \\
\text { „Untersuchungskomitees» (Sledstwennyj komitet) zu prüfen. }\end{array}$ \\
\hline 5.3.2007 & $\begin{array}{l}\text { In Moskau legt eine Delegation der KPRF unter Führung von Gennadij Sjuganow anlässlich des } 55 \text {. Todestages Sta- } \\
\text { lins Blumen an seinem Grab an der Kremlmauer nieder. An der Veranstaltung nehmen } 400 \text { Personen teil. }\end{array}$ \\
\hline 5.3 .2007 & $\begin{array}{l}\text { Maksim Resnik, der inhaftierte Petersburger Jabloko-Politiker, tritt in Hungerstreik, um gegen die gegen ihn erho- } \\
\text { benen Beschuldigungen zu protestieren. }\end{array}$ \\
\hline 5.3 .2007 & $\begin{array}{l}\text { Gazprom nimmt die Gaslieferungen an die Ukraine in vollem Umfang wieder auf. Die Verhandlungen mit der } \\
\text { ukrainischen Seite laufen indes weiter. }\end{array}$ \\
\hline 6.3 .2007 & $\begin{array}{l}\text { Die Zeitschrift „Forbes" publiziert ihre neue Liste der reichsten Leute der Welt. Unter 1.125 Milliardären sind } 469 \\
\text { US-Amerikaner, } 87 \text { Russen, } 66 \text { Chinesen (40 Volksrepublik, } 26 \text { Hongkong) und } 59 \text { Deutsche. An der Spitze der } \\
\text { russischen Milliardäre stehen: Oleg Deripaska (40 Jahre, 28,0 Mrd.), Roman Abramowitsch (41 Jahre, 23,5 Mrd.), } \\
\text { Aleksei Mordaschow (42 Jahre, 21,2 Mrd.), Michail Fridman (43 Jahre, 20,8 Mrd.), Wladimir Lisin, (51 Jahre, } \\
20,3 \text { Mrd.), Michail Prochorow (42 Jahre, 19,5 Mrd.), Wladimir Potanin (47 Jahre, 19,3 Mrd.) und Suleiman } \\
\text { Kerimow (42 Jahre, 17,5 Mrd.) }\end{array}$ \\
\hline 6.3 .2007 & $\begin{array}{l}\text { Ramzan Kadyrow, der Präsident Tschetscheniens, wird aus dem Verband der Journalisten ausgeschlossen, nach- } \\
\text { dem eine Überprüfung ergeben hat, dass es keinen Beleg für eine einschlägige Tätigkeit Kadyrows gibt. Die tsche- }\end{array}$ \\
\hline
\end{tabular}

Die Russland-Analysen werden mit Unterstützung durch die Otto-Wolff-Stiftung gemeinsam von der Forschungsstelle Osteuropa an der Universität Bremen und der Deutschen Gesellschaft für Osteuropakunde herausgegeben.

Die Meinungen, die in den Russland-Analysen geäußert werden, geben ausschließlich die Auffassung der Autoren wieder. Abdruck und sonstige publizistische Nutzung sind nach Rücksprache mit der Redaktion gestattet. Redaktion und technische Gestaltung: Matthias Neumann, Heiko Pleines, Hans-Henning Schröder Russland-Analysen-Layout: Cengiz Kibaroglu ISSN 1613-3390 (c) 2008 by Forschungsstelle Osteuropa, Bremen

Forschungsstelle Osteuropa • Publikationsreferat • Klagenfurter Str. 3 • 28359 Bremen • Telefon: +49 421-218-7891 • Telefax: +49 421-218-3269 e-mail: publikationsrefera@@osteuropa.uni-bremen.de • Internet-Adresse: http://www.laender-analysen.de/russland/ 


\section{Lesehinweis}

\section{Kostenlose E-Mail-Dienste der Forschungsstelle Osteuropa auf www.laender-analysen.de}

\section{Russland-Analysen}

Die »Russlandanalysen« bieten wöchentlich eine Kurzanalyse zu einem aktuellen Thema, ergänzt um Grafiken und Tabellen. Zusätzlich gibt es eine Wochenchronik aktueller politischer Ereignisse.

Abonnement unter: fsopr@uni-bremen.de

\section{Russian Analytical Digest}

Der Russian Analytical Digest bietet zweimal monatlich englischsprachige Kurzanalysen sowie illustrierende Daten zu einem aktuellen Thema.

Abonnement unter: http:// w w w.res.ethz.ch/ a nalysis/ rad/

\section{kultura. Russland-Kulturanalysen}

Die Russland-Kulturanalysen diskutieren in kurzen, wissenschaftlich fundierten, doch publizistisch-aufbereiteten Beiträgen signifikante Entwicklungen der Kultursphäre Russlands. Jede Ausgabe enthält zwei Analysen und einige Kurztexte bzw. Illustrationen. Erscheinungsweise: monatlich, in je einer deutschen und englischen Ausgabe.

Abonnement unter: fsopr@uni-bremen.de

\section{Ukraine-Analysen}

Die Ukraine-Analysen bieten zweimal monatlich eine Kurzanalyse zu einem aktuellen Thema aus Politik, Wirtschaft oder Kultur, ergänzt um Grafiken und Tabellen. Zusätzlich gibt es eine Chronik aktueller Ereignisse.

Abonnement unter: fsopr@uni-bremen.de

\section{Polen-Analysen}

Die Polen-Analysen bieten zweimal monatlich eine Kurzanalyse zu einem aktuellen Thema aus Politik, Wirtschaft oder Kultur, ergänzt um Grafiken und Tabellen. Zusätzlich gibt es eine Chronik aktueller Ereignisse.

Abonnement unter: http:// w w w.polen-analysen.de

\section{Zentralasien-Analysen}

Die Zentralasien-Analysen bieten monatlich eine Kurzanalyse zu einem aktuellen Thema aus Politik, Wirtschaft oder Kultur, ergänzt um Grafiken und Tabellen. Zusätzlich gibt es eine Chronik aktueller Ereignisse.

Abonnement unter: zentralasien-a na lysen@ dgo -online.org

\section{Bibliographische Dienste}

Die vierteljährlich erscheinenden Bibliographien informieren über englisch- und deutschsprachige Neuerscheinungen zu Polen, Russland, Tschechischer und Slowakischer Republik, Ukraine sowie zu den zentralasiatischen und kaukasischen Staaten. Erfasst werden jeweils die Themenbereiche Politik, Außenpolitik, Wirtschaft und Soziales.

Abonnement unter: fsopr@uni-bremen.de 